\title{
A transcriptomic analysis of Chrysanthemum nankingense provides insights into the basis of low temperature tolerance
}

\author{
Liping Ren ${ }^{1,2}$, Jing Sun ${ }^{1}$, Sumei Chen ${ }^{1}$, Jiaojiao Gao ${ }^{1}$, Bin Dong ${ }^{1}$, Yanan Liu ${ }^{1}, X_{i a o l o n g}$ Xia ${ }^{1}$, Yinjie Wang ${ }^{1}$, \\ Yuan Liao ${ }^{1}$, Nianjun Teng ${ }^{1}$, Weimin Fang ${ }^{1}$, Zhiyong Guan ${ }^{1}$, Fadi Chen ${ }^{1,2^{*}}$ and Jiafu Jiang ${ }^{1 *}$
}

\begin{abstract}
Background: A major constraint affecting the quality and productivity of chrysanthemum is the unusual period of low temperature occurring during early spring, late autumn, and winter. Yet, there has been no systematic investigation on the genes underlying the response to low temperature in chrysanthemum. Herein, we used RNA-Seq platform to characterize the transcriptomic response to low temperature by comparing different transcriptome of Chrysanthemum nankingense plants and subjecting them to a period of sub-zero temperature, with or without a prior low temperature acclimation.
\end{abstract}

Results: Six separate RNA-Seq libraries were generated from the RNA samples of leaves and stems from six different temperature treatments, including one cold acclimation (CA), two freezing treatments without prior CA, two freezing treatments with prior CA and the control. At least seven million clean reads were obtained from each library. Over $77 \%$ of the reads could be mapped to sets of $C$. nankingense unigenes established previously. The differentially transcribed genes (DTGs) were identified as low temperature sensing and signalling genes, transcription factors, functional proteins associated with the abiotic response, and low temperature-responsive genes involved in post-transcriptional regulation. The differential transcription of 15 DTGs was validated using quantitative RT-PCR.

Conclusions: The large number of DTGs identified in this study, confirmed the complexity of the regulatory machinery involved in the processes of low temperature acclimation and low temperature/freezing tolerance.

Keywords: Transcriptome, RNA Sequencing, Low temperature tolerance, Ornamental plant

\section{Background}

Chrysanthemum (Chrysanthemum morifolium) is a popular ornamental plant worldwide [1,2]. Chrysanthemum plants are susceptible to damage when exposed to prolonged periods of low temperature; therefore, improving their tolerance to cold stress is perceived as an important breeding goal. The high chromosome number and polyploidy of major ornamental species complicate the genetics and capacity for gene discovery [3]. Hence, $C$. nankingense has been considered as a convenient genomic model due to its simple diploid nature. In addition, it

\footnotetext{
* Correspondence: chenfd@njau.edu.cn; jiangjiafu@njau.edu.cn ${ }^{1}$ College of Horticulture, Nanjing Agricultural University, Nanjing 210095, China

${ }^{2}$ Jiangsu Province Engineering Lab for Modern Facility Agriculture Technology \& Equipment, No. 1 Weigang, Nanjing 210095, Jiangsu Province, China
}

displays better tolerance to low temperature as compared to the ornamental polyploid species [4].

Temperature is a major determinant of the geographical distribution and length of the growing season in most plant species [5,6]. However, episodes of low temperature during the growing season cause a substantial loss in the yield of many temperate crops. During chrysanthemum production, specifically in China, extreme low temperatures in early spring and winter, unusual freezing temperatures during late cold spring, and sudden frosts in fall often lead to growth arrest and block flower buds or inflorescence, which in turn result in significant economic losses every year [7]. Temperate plant species can acquire the ability to withstand a prolonged period of sub-zero temperature if they are previously exposed to a period of low temperature above $0^{\circ} \mathrm{C}$; this phenomenon is known as low temperature
C Biomed Central

(c) 2014 Ren et al.; licensee BioMed Central Ltd. This is an Open Access article distributed under the terms of the Creative Commons Attribution License (http://creativecommons.org/licenses/by/4.0), which permits unrestricted use, distribution, and reproduction in any medium, provided the original work is properly credited. The Creative Commons Public Domain Dedication waiver (http://creativecommons.org/publicdomain/zero/1.0/) applies to the data made available in this article, unless otherwise stated. 
acclimation $[5,8,9]$. However, the molecular basis of cold acclimation (CA) and low temperature/freezing tolerance in chrysanthemum has not yet been explored. In the present study, we aim to fish out candidate genes underlying the process of $\mathrm{CA}$ and response to low /freezing temperature, which will help to elucidate the molecular basis of the cold response in C. nankingense, and then improve the chrysanthemum varieties cold tolerance.

In Arabidopsis thaliana and some of the winter cereals, a variety of physiological, biochemical, and molecular changes are known to occur during the low temperature acclimation process [9]. In these species, the physical state of the plasma membrane has been shown to be an important determinant of the plant's ability to sense changes in the air temperature [10-14]. Membrane rigidification leads to an increase in the cytosolic concentration of the $\mathrm{Ca}^{2+}$ ion [15], which is regarded as a major regulator of low temperature responsive factor. Certain $\mathrm{Ca}^{2+}$-dependent protein kinases have also been recognized as positive regulators [16]. The mitogen-activated protein kinase (MAPK) cascade participates in the low temperature signalling and low temperature tolerance [17]. Three candidate chilling response genes, encoding MAPKKK (MAPK kinase kinase), CLC-D (chloride channel D) and RLK (receptor-like protein kinase) homologues are all up-regulated following chilling stress in Maize [18]. The CBF low temperatureresponse pathway is well conserved across a diversity of species and is a significant component of tolerance to sub-zero temperatures $[7,19]$. The CBF pathway is positively regulated by the circadian clock components CCA1 and LHY [20], while the INDUCER OF CBF EXPRESSION 1 (ICE1) protein acts upstream of CBF in the low temperature response pathway [21]. A high expression of osmotically responsive gene (HOS) 1 acts as a negative regulator of the low temperature response $[21,22]$. Salicylic acid (SA) is also involved in the response to low temperature stress [23,24]. RNA processing and nucleocytoplasmic transport play crucial roles in plant stress [25].

The RNA-Seq platform has become a key technology for quantifying the transcriptional response in nonmodel organisms or those with genome characteristics extremely difficult to whole-genome sequencing [26,27]. In tea (Camellia sinensis), this approach has enabled the recognition of 1,770 differentially transcribed genes (DTGs) induced during low temperature acclimation [28]. A similar transcriptomic analysis of Jatropha curcas identified over 3,000 genes as being up- or down-regulated by low temperature [29]. While in Anthurium sp., the method of digital gene expression enabled 39 low temperature-inducible transcription factors (TFs) to be identified [30].

Here, the RNA-Seq platform based on Illumina NGS technology was used to characterize the transcriptomic response to low temperature by comparing the different transcriptome of $C$. nankingense plants subjected to periods of sub-zero temperature with or without a prior low temperature acclimation, with a view to gaining a deeper insight into the molecular basis of this physiological adaptation. In addition, the identified candidate genes will be useful for improving adaptation to low temperature and enhancing productivity and geographical distribution.

\section{Results}

\section{RNA-Seq libraries and reads mapping}

An overview of the RNA-Seq reads derived from the six libraries using Illumina $\mathrm{HiSeq}^{\mathrm{m}} 2000$ platform is presented in Table 1 and Additional file 1: Figure S1. The raw sequence data have been deposited in the NCBI Sequence Read Archive (http://trace.ncbi.nlm.nih.gov/Traces/sra_sub/ sub.cgi). The number of clean reads per library ranged from 7.01 to 7.47 million, and the total number of nucleotides sequenced from 343,646,114 to 365,975,267 (Accession No. for library A SRS591717; Accession No. for library B1 591719; Accession No. for library B2 591720;

Table 1 Summary of mapping result

\begin{tabular}{|c|c|c|c|c|c|c|c|c|}
\hline $\begin{array}{l}\text { Sample } \\
\text { ID }\end{array}$ & Total reads & $\begin{array}{l}\text { Total base } \\
\text { pairs }\end{array}$ & $\begin{array}{l}\text { Total mapped } \\
\text { reads }\end{array}$ & $\begin{array}{l}\text { Perfect } \\
\text { match }\end{array}$ & $\begin{array}{l}<=2 \text { bp } \\
\text { Mismatch }\end{array}$ & $\begin{array}{l}\text { Unique } \\
\text { match }\end{array}$ & $\begin{array}{l}\text { Multi-position } \\
\text { match }\end{array}$ & $\begin{array}{l}\text { Total unmapped } \\
\text { reads }\end{array}$ \\
\hline$A$ & $\begin{array}{l}7270059 \\
(100.00 \%)\end{array}$ & $\begin{array}{l}356232891 \\
(100.00 \%)\end{array}$ & $\begin{array}{l}5627806 \\
(77.41 \%)\end{array}$ & $\begin{array}{l}4003637 \\
(55.07 \%)\end{array}$ & $\begin{array}{l}1624169 \\
(22.34 \%)\end{array}$ & $\begin{array}{l}4190635 \\
(57.64 \%)\end{array}$ & $1437171(19.77 \%)$ & $1642253(22.59 \%)$ \\
\hline B1 & $\begin{array}{l}7052023 \\
(100.00 \%)\end{array}$ & $\begin{array}{l}345549127 \\
(100.00 \%)\end{array}$ & $\begin{array}{l}5480579 \\
(77.72 \%)\end{array}$ & $\begin{array}{l}3893154 \\
(55.21 \%)\end{array}$ & $\begin{array}{l}1587425 \\
(22.51 \%)\end{array}$ & $\begin{array}{l}4099659 \\
(58.13 \%)\end{array}$ & 1380920 (19.58\%) & 1571444 (22.28\%) \\
\hline B2 & $\begin{array}{l}7013186 \\
(100.00 \%)\end{array}$ & $\begin{array}{l}343646114 \\
(100.00 \%)\end{array}$ & $\begin{array}{l}5444098 \\
(77.63 \%)\end{array}$ & $\begin{array}{l}3862298 \\
(55.07 \%)\end{array}$ & $\begin{array}{l}1581800 \\
(22.55 \%)\end{array}$ & $\begin{array}{l}4128240 \\
(58.86 \%)\end{array}$ & 1315858 (18.76\%) & $1569088(22.37 \%)$ \\
\hline C1 & $\begin{array}{l}7228380 \\
(100.00 \%)\end{array}$ & $\begin{array}{l}354190620 \\
(100.00 \%)\end{array}$ & $\begin{array}{l}5857437 \\
(81.03 \%)\end{array}$ & $\begin{array}{l}4335313 \\
(59.98 \%)\end{array}$ & $\begin{array}{l}1522124 \\
(21.06 \%)\end{array}$ & $\begin{array}{l}4401306 \\
(60.89 \%)\end{array}$ & $1456131(20.14 \%)$ & 1370943 (18.97\%) \\
\hline $\mathrm{C} 2$ & $\begin{array}{l}7299665 \\
(100.00 \%)\end{array}$ & $\begin{array}{l}357683585 \\
(100.00 \%)\end{array}$ & $\begin{array}{l}5629870 \\
(77.13 \%)\end{array}$ & $\begin{array}{l}3989898 \\
(54.66 \%)\end{array}$ & $\begin{array}{l}1639972 \\
(22.47 \%)\end{array}$ & $\begin{array}{l}4215854 \\
(57.75 \%)\end{array}$ & 1414016 (19.37\%) & 1669795 (22.87\%) \\
\hline CK & $\begin{array}{l}7468883 \\
(100.00 \%)\end{array}$ & $\begin{array}{l}365975267 \\
(100.00 \%)\end{array}$ & $\begin{array}{l}5865590 \\
(78.53 \%)\end{array}$ & $\begin{array}{l}4203842 \\
(56.28 \%)\end{array}$ & $\begin{array}{l}1661748 \\
(22.25 \%)\end{array}$ & $\begin{array}{l}4403996 \\
(58.96 \%)\end{array}$ & 1461594 (19.57\%) & 1603293 (21.47\%) \\
\hline
\end{tabular}


Accession No. for library C1 591721; Accession No. for library C2 591722; Accession No. for library CK 591679). The proportion of clean reads was over $99 \%$ in each library (Additional file 1: Figure S1). Overall, from library A to library CK, 7,270,059, 7,052,023, 7,013,186, 7,228,380, $7,299,665$, and $7,468,883$ clean reads were obtained respectively. Correspondingly, 356,232,891, 345,549,127, $343,646,114,354,190,620,357,683,585$, and $365,975,267$ total base-pairs (Table 1) were generated. The clean reads were mapped onto a reference gene database, which included all known $C$. nankingense unigene sequences. Raw sequence data were deposited in the NCBI Sequence Read Archive database under the accession number SRP041330. The proportion of unambiguously mapped reads per library ranged from $77.13 \%$ in library $\mathrm{C} 2$ to $81.03 \%$ (C1), and the proportion of unique matches from $57.64 \%$ (A) to $60.89 \%$ (C1). As the number of reads increased, the identification rate of new genes slowed, which indicated the saturation around seven million reads (7,013,186 in library B2, 7,468,883 in CK) (Additional file 2: Figure S2).

\section{Quantification of transcripts and identification of differentially transcribed genes (DTGs)}

The quality of the RNA-Seq dataset is assessed by gene coverage, which is the percentage of a gene covered by reads. This value is determined as the ratio of the base number in a gene covered by unique mapping reads to the total bases number of that gene. The distribution of the six libraries was presented in Additional file 3: Figure S3. In addition, transcript abundances for each gene (Additional file 4: Table S1) were calculated according to the method following Mortazavi et al. [31]. Moreover, differential transcription was identified through pairwise comparison between various libraries, by setting a threshold FDR of 0.001 and a $\mid \log 2$ ratio $\mid$ of 1 based on the algorithm developed by Audic et al. [32]. From the seven comparisons, including treatment CKA (CK vs A), CKB1 (CK vs B1), CKB2 (CK vs B2), CKC1 (CK vs C1), $\mathrm{CKC} 2$ (CK vs $\mathrm{C} 2$ ), $\mathrm{AC} 1(\mathrm{~A}$ vs $\mathrm{C} 1$ ), and $\mathrm{AC} 2(\mathrm{~A}$ vs $\mathrm{C} 2)$, the results showed that a large number of DTGs were identified (Additional files 5, 6, 7, 8, 9, 10 and 11: Table S2-8). The number of DTGs detected was as follows: treatment CKA, 3,779 (2,096 up- and 1,683 down-regulated); CKB1, 337 (250 and 87); CKB2, 718 (571 and 147); CKC1, 3,722 (2,271 and 1,451); CKC2, 4,119 (2,611 and 1,508); AC1, 194 (169 and 25); and AC2, 111 (92 and 19) (Figure 1). These results indicated that more DTGs were identified in the treatments, which underwent a prior CA (A, C1 and $\mathrm{C} 2$ ), as compared to the treatments which didn't undergo $\mathrm{CA}$ (B1 and B2). In addition, a smaller number of DTGs was found in $\mathrm{A} v s \mathrm{C} 1$ and $\mathrm{A}$ vs $\mathrm{C} 2$ comparisons than in CK vs B1 and CK vs B2. Moreover, on extending the freezing treatment, fewer DTG were detected in A vs $\mathrm{C} 2$ comparison than in $\mathrm{A}$ vs $\mathrm{C} 1$. However, contrary results were obtained in case of $\mathrm{CK} v s$ B1 and CK vs B2 comparisons. Based on the assumption that genes with similar expression patterns usually exhibit functional correlation, the consistency of the DTGs was checked by multiple comparisons clustering among the CKA, CKC1 and CKC2; between the CKB1 and CKB2 treatments; and between the comparisons, $\mathrm{A} v s \mathrm{C} 1$ and $\mathrm{A} v s \mathrm{C} 2$. A total of 2,340 DTGs were observed in the first multiple comparison clustering, out of which, only three genes behaved inconsistently (that is, showed up-regulation in one treatment and down-regulation in the other, or vice versa). Of the 2,337 consistent DTGs, 1,410 were upand 927 were down-regulated (Additional file 12: Table S9). In the second multiple comparison clustering, 188 DTGs (142 up- and 46 down-regulated) were obtained (Additional file 13: Table S10), and all were found to be consistent. In the third comparison clustering between

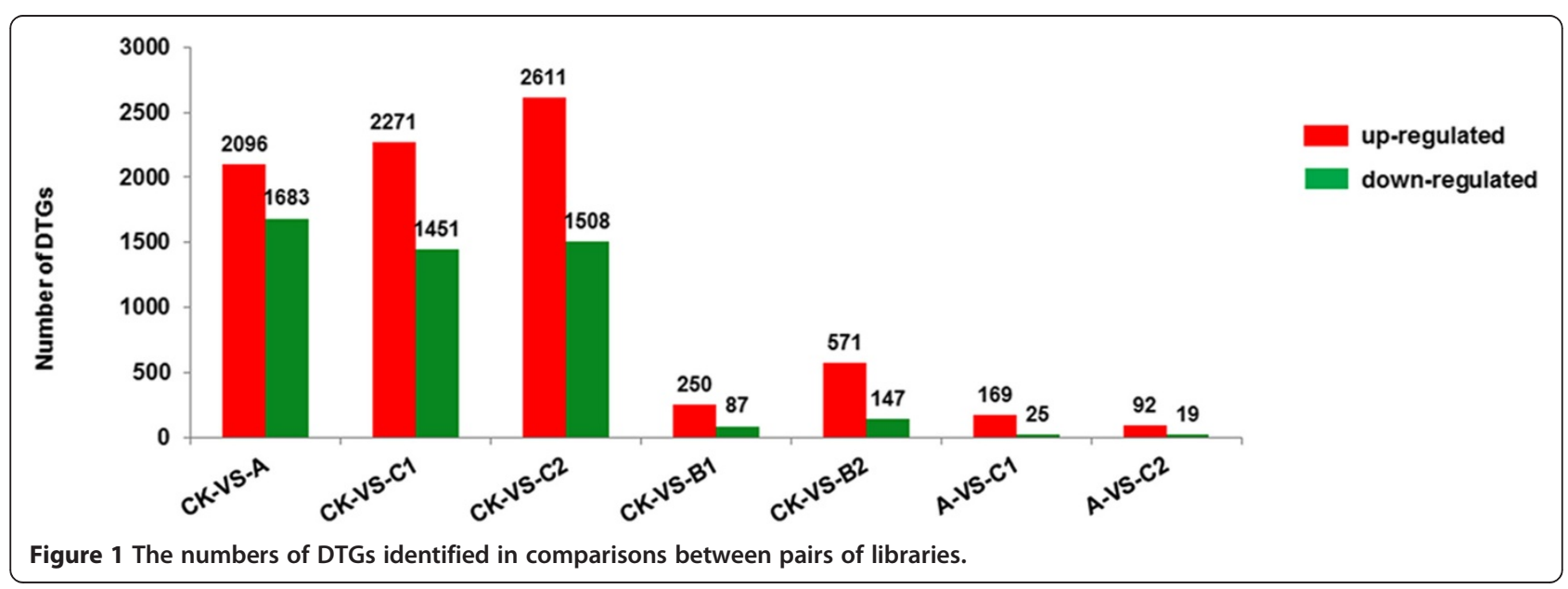


A vs $\mathrm{C} 1$ and $\mathrm{A}$ vs $\mathrm{C} 2,38$ DTGs (37 up- and 1 downregulated) showed consistency (Additional file 14: Table S11).

\section{GO classification of differentially transcribed genes}

In the treatment CKA, 1,535 of the 3,779 DTGs could be assigned a GO term; the equivalent number for other comparisons were as follows: treatment CKB1, 155/337; CKB2, 246/718; CKC1, 1,522/3722; CKC2, 1,691/4119; A vs $\mathrm{C} 1,60 / 194$; and $\mathrm{A}$ vs $\mathrm{C} 2,30 / 111$ (Additional file 15: Table S12). For CK vs A, 21 GO classes fell into the categories "biological process", 12 into "cellular component" and 11 into "molecular function". The equivalent distribution in CK vs B1 was 18, 10, and 7; in CK vs B2, 20, 11 and 9; in both, CK vs C1 and CK vs C2, 21, 12, and 11; in $\mathrm{A}$ vs $\mathrm{C} 1,17,9$, and 5; and in $\mathrm{A}$ vs $\mathrm{C} 2,11,7$, and 5. The major classes of biological process among the DTGs in CK vs A comparison were "metabolic process", "cellular process", "single organism process", "response to stimulus", "localization", "establishment localization", "biological regulation" and "regulation of biological process"; the predominant cellular components were "cell", "cell part", "organelle" and "organelle part"; and for molecular function "binding", "catalytic activity", "transporter activity", "nucleic acid binding transcription factor activity" and "antioxidant activity". Only a few genes belonged to the categories "cell killing", "electron carrier activity", "positive regulation of biological process", "extracellular matrix", "receptor activity", "cell junction", "protein binding transcription factor activity" and "carbon utilization". The details of GO classification of DTGs in CK vs A, and other comparisons are presented in Figure 2. Plant hormone signal transduction pathways (mediated by either auxin or gibberellin) were well represented, particularly those associated with auxin-mediated signalling. Low temperature sensing and signalling genes influenced by $\mathrm{Ca}^{2+}$, as well as other protein kinases were also identified. A number of TF families, genes encoding functional proteins and posttranslational regulated genes were represented.

\section{Function annotation of DTGs using the KEGG database}

Unigene KEGG annotation was aimed at DTGs from the above comparisons. In the CK vs A comparison, 1,972 DTGs were assigned to the KEGG database involved 122 pathways; for CK vs B1, 175 DTGs were assigned to 61 pathways; for CK vs B2, 302 DTGs were assigned to 75 pathways; for B1 vs B2, 100 DTGs were assigned to 46 pathways; for CK vs C1, 1,915 DTGs were assigned to 120 pathways; for CK vs C2, 2,175 DTGs were assigned to 120 pathways; for A vs C1, 90 DTGs were assigned to 46 pathways; and for A vs C2, 37 DTGs were assigned to 29 pathways. The details of the KEGG classification of the above comparisons are presented in Additional file 16: Table S13. The major pathways identified were "metabolism",

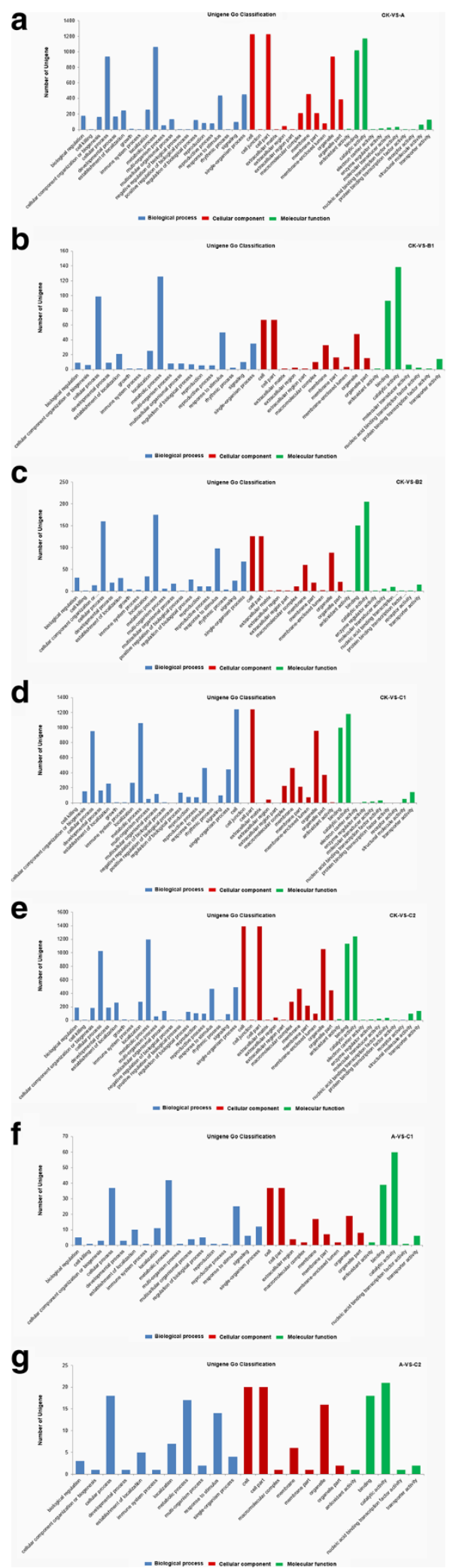


Figure 2 Gene Ontology (GO) classification of the DTGs identified in each comparison between a pair of libraries. DTGs were annotated in three categories: biological process, cellular component and molecular function. Y-axis (right) represents the number of DTGs in each category; Y-axis (left) represents the percentage of a specific category of DTGs within that main category. Panels $\mathbf{a}, \mathbf{b}, \mathbf{c}, \mathbf{d}, \mathbf{e}$, $\mathbf{f}$ and $\mathbf{g}$ (left) represents DTGs in the comparison between library CK $\left(22^{\circ} \mathrm{C}\right)$ and $\mathrm{A}\left(4^{\circ} \mathrm{C}\right.$ for one week) (CK-VS-A) (right), library CK and B1 ( $-5^{\circ} \mathrm{C}$ for $\left.1 \mathrm{~h}\right)$ (CK-VS-B1) (right), library CK and B2 $\left(-5^{\circ} \mathrm{C}\right.$ for $\left.2 \mathrm{~h}\right)$ (CK-VS-B2) (right), library $\mathrm{CK}$ and $\mathrm{C} 1\left(4^{\circ} \mathrm{C}\right.$ for one week, followed by $-5^{\circ} \mathrm{C}$ for $\left.1 \mathrm{~h}\right)$ (CK-VS-C1) (right), library CK and $\mathrm{C} 2\left(4^{\circ} \mathrm{C}\right.$ for one week, followed by $-5^{\circ} \mathrm{C}$ for $2 \mathrm{~h}$ ) (CK-VS-C2) (right), library A and C1 (A-VS-C1) (right), and library A and C2 (A-VS-C2) (right) respectively.

"biosynthesis of secondary metabolites", "hormone signal transduction", "plant-pathogen interaction", "spliceosome", "phenylpropanoid biosynthesis", "endocytosis" and "protein processing in the endoplasm", "ribosome" and "starch and sucrose metabolism".

\section{Genes involved in the response to low temperature}

More DTGs were identified in the CK vs A, CK vs C1 and $\mathrm{CK}$ vs $\mathrm{C} 2$ comparisons than in the $\mathrm{CK} v s \mathrm{~B} 1$ and $\mathrm{CK}$ vs $\mathrm{B} 2$. This observation demonstrated that many genes were involved in low temperature acclimation. The DTGs revealed in the comparisons, $\mathrm{A} v s \mathrm{C} 1$ and $\mathrm{A} v s \mathrm{C} 2$, were found to be involved in response to freezing treatment, when the plants were exposed to prior CA. However, the DTGs obtained from the $\mathrm{CK}$ vs $\mathrm{B} 1$ and $\mathrm{CK}$ vs $\mathrm{B} 2$ comparisons participated in the response mechanism, when the plants didn't undergo prior CA. Some of all the DTGs belonged to the group of genes involved in cold sensing and signal transduction pathways. $\mathrm{Ca}^{2+}$ signaling pathway, which is regarded as an important signal sensing and transduction pathway under stresses, includes many members, such as cation/calcium exchangers (CCX), calcium-binding proteins $(\mathrm{CBP})$, calmodulin-like proteins (CML), CBLinteracting protein kinases (CIPK), calcium-dependent protein kinases (CDPK) and calmodulin-binding receptorlike kinases (CBRLK). The CK vs A comparison included three $C C X$, two $C B P$, five $C M L$, three $C I P K$, and one $C B R L K$ genes; the equivalent order for the CK vs C1 comparison was, respectively, three, one, five, five and one; for $\mathrm{CK}$ vs $\mathrm{C} 2$, three, zero, four, four and one; for CK vs B1, zero, one, three, zero and zero, along with one sodium/ calcium exchanger family protein gene (SCE); for CK $v s$ B2, zero, zero, five, two, zero, along with one SCE and one CDPK; for A vs C1, zero, one, two, one, zero; and for $\mathrm{A}$ vs $\mathrm{C} 2$, only one CBP was involved. In the comparisons, CK vs A, CK vs C1, and CK vs C2, three CCX (CL8580.Contig_S2_3, Unigene82641_S2_3, and Unigene21268_S2_3), one CML (Unigene74966_S2_1), and one CDPK (Unigene5836_S2_1) were all found to be upregulated; in addition, two CMLs (Unigene13572_S2_1 and 3462_S2_3), and one CDPK (CL8813.Contig2_S2_1) show- ed down-regulation. One CML (Unigene76667_S2_3) was found to be up-regulated in both comparisons, CK vs B1 and $\mathrm{CK}$ vs B2. These findings indicated that the genes related to $\mathrm{Ca}^{2+}$ signaling pathway played an essential role in the phenomenon of $\mathrm{CA}$ and freezing response without a prior CA; the complete details of DTGs involved in $\mathrm{Ca}^{2+}$ signalling pathway are presented in Table 2. No DTGs associated with the MAPK cascade was identified in the CK vs A comparison. In contrast, one MAPKKK and one $M A P K$ gene were found to be differentially transcribed in $\mathrm{CK}$ vs $\mathrm{C} 1$, and one $M A P K K K$ was represented in CK vs $\mathrm{C} 2$ as well as in CK vs B1. Four MAPKKK genes were observed in the CK vs $\mathrm{B} 2$ comparison, and one MAPKKK gene was identified in both, $\mathrm{A} v s \mathrm{C} 1$ and $\mathrm{A} v s \mathrm{C} 2$. The results showed that MAPK cascade, especially MAPKKK genes, participated in the plant's response to freezing treatment, instead of CA. Therefore, all the DTGs involved in MAPK cascade were found to be up-regulated (Table 3). A higher number of genes encoding serine/threonine-protein kinases were differentially transcribed in CK vs $\mathrm{A}$, CK vs $\mathrm{C} 1$ and $\mathrm{CK}$ vs $\mathrm{C} 2$ than in CK vs B1 or CK vs B2 (Table 4). However, no DTG encoding this specific kinase was identified in the $\mathrm{A} v s \mathrm{C} 1$ or $\mathrm{A} v s \mathrm{C} 2$ comparisons.

In the present study, members of various low temperature-responsive transcription factor (TF) families were identified; and 43, 44 and 46 such genes were found to be differentially transcribed in the comparisons, CK vs A, CK vs $\mathrm{C} 1$ and $\mathrm{CK}$ vs $\mathrm{C} 2$, respectively. The major TF families presented were AP2/ERF, bHLH, WRKY and TCP, along with small numbers of MYB, MYC, NAC, DOF, and the trihelix family. The CK vs B1 and CK vs B2 comparisons showed 8 and 19 TF DTGs, respectively. The number of TF DTGs identified in the $\mathrm{CK}$ vs $\mathrm{B} 1$ or $\mathrm{CK}$ vs $\mathrm{B} 2$ comparison was lesser than the comparisons, $\mathrm{CK}$ vs $\mathrm{A}, \mathrm{CK}$ vs $\mathrm{C} 1$, or $\mathrm{CK}$ vs $\mathrm{C} 2$, which were involved in the process of low temperature acclimation. A larger number of TF DTGs were present in CK vs B2 than in the CK vs B1 comparison. In addition, five (3 WRKYs, 1 DREB and 1 ERF) and seven TF DTGs (1 DREB, 1 bHLH and 5 ERFs) were found in the $\mathrm{A} v s \mathrm{C} 1$ and $\mathrm{A}$ vs $\mathrm{C} 2 \mathrm{com}-$ parisons, respectively. The identified TF DTGs from the A vs $\mathrm{C} 1$ and $\mathrm{A}$ vs $\mathrm{C} 2$ comparisons, were involved in response to freezing treatment in plants with prior exposure to CA. The differently transcribed TFs of the CKA treatment, the $\mathrm{A}$ vs $\mathrm{C} 1$, and $\mathrm{A}$ vs $\mathrm{C} 2$ comparisons are presented in Table 5 .

Other relevant classes of protein, which featured as DTGs products, were dehydrin, LEA (late embryogenesis abundant) proteins, heat shock proteins (HSPs). The proteins involved in post-transcriptional regulation, such as ribosomal proteins and a DEAD-box ATP-dependent RNA helicase $(\mathrm{RH})$, were particularly found in the CK vs $\mathrm{A}, \mathrm{CK}$ vs $\mathrm{C} 1$ and $\mathrm{CK}$ vs $\mathrm{C} 2$ comparisons. Yet, with respect to the above classes of proteins, only one LEA was 
Table 2 The differential gene expression of genes involved in $\mathrm{Ca}^{2+}$ signalling pathway in each comparison

\begin{tabular}{|c|c|c|c|c|c|c|}
\hline Comparison & GenelD & $\begin{array}{l}\log 2 \\
\text { ratio }\end{array}$ & $\begin{array}{l}\text { Up-Down- } \\
\text { Regulation }\end{array}$ & P-value & FDR & Gene description \\
\hline \multirow[t]{14}{*}{ CK vs A } & CL8580.Contig1_S2_3 & 12.34 & Up & $1.38 \mathrm{E}-17$ & $6.12 \mathrm{E}-16$ & cation/calcium exchanger4 \\
\hline & Unigene82641_S2_3 & 5.43 & Up & $3.53 \mathrm{E}-12$ & $1.11 \mathrm{E}-10$ & cation/calcium exchanger 4-like \\
\hline & Unigene21268_S2_3 & 4.98 & Up & $9.77 \mathrm{E}-17$ & 4.15E-15 & cation/calcium exchanger 4 \\
\hline & Unigene19284_S2_1 & -1.46 & Down & 5.67E-05 & 0.000728 & calcium-binding protein \\
\hline & Unigene27711_S2_3 & -1.30 & Down & 4.81E-30 & $3.78 \mathrm{E}-28$ & calcium-binding allergen Ole e 8-like \\
\hline & Unigene74966_S2_1 & 1.09 & Up & 2.72E-08 & $5.82 \mathrm{E}-07$ & calmodulin-like protein 1-like \\
\hline & Unigene13572_S2_1 & -2.40 & Down & $1.22 \mathrm{E}-33$ & 1.07E-31 & calmodulin-like protein 5-like \\
\hline & Unigene3462_S2_3 & -5.71 & Down & $3.14 \mathrm{E}-15$ & $1.22 \mathrm{E}-13$ & calcium-binding protein \\
\hline & Unigene76667_S2_3 & -1.46 & Down & $5.29 \mathrm{E}-13$ & $1.77 \mathrm{E}-11$ & calcium-binding protein CML24-like \\
\hline & Unigene42277_S2_1 & -1.40 & Down & $1.82 \mathrm{E}-09$ & 4.44E-08 & calcium-binding protein CML45-like \\
\hline & Unigene86355_S2_3 & 1.00 & Up & 3.93E-06 & 0.000062 & $\begin{array}{l}\text { calmodulin-binding receptor-like } \\
\text { cytoplasmic kinase 3-like }\end{array}$ \\
\hline & Unigene5836_S2_1 & 2.01 & Up & $1.42 \mathrm{E}-44$ & 1.69E-42 & $\begin{array}{l}\text { CBL-interacting serine/threonine-protein } \\
\text { kinase }\end{array}$ \\
\hline & CL4456.Contig1_S2_3 & 1.07 & Up & $2.72 \mathrm{E}-93$ & 7.05E-91 & $\begin{array}{l}\text { CBL-interacting serine/threonine- } \\
\text { protein kinase 6-like isoform } 1\end{array}$ \\
\hline & CL8813.Contig2_S2_1 & -2.63 & Down & $6.68 \mathrm{E}-23$ & $3.94 \mathrm{E}-21$ & $\begin{array}{l}\text { CBL-interacting serine/threonine- } \\
\text { protein kinase } 25 \text {-like }\end{array}$ \\
\hline \multirow[t]{15}{*}{ CK vs C1 } & CL8580.Contig1_S2_3 & 12.35 & Up & $6.77 \mathrm{E}-18$ & $3.12 \mathrm{E}-16$ & cation/calcium exchanger 4-like \\
\hline & Unigene82641_S2_3 & 5.32 & Up & $1.92 \mathrm{E}-11$ & $5.60 \mathrm{E}-10$ & cation/calcium exchanger 4-like \\
\hline & Unigene21268_S2_3 & 4.56 & Up & $2.22 \mathrm{E}-12$ & 7.00E-11 & cation/calcium exchanger 4 \\
\hline & Unigene75028_S2_1 & 1.30 & Up & $2.91 \mathrm{E}-07$ & $5.47 \mathrm{E}-06$ & calcium-binding protein PBP1-like \\
\hline & Unigene74966_S2_1 & 1.37 & Up & $1.43 \mathrm{E}-13$ & 4.94E-12 & calmodulin-like protein 1-like \\
\hline & Unigene86214_S2_3 & 1.20 & Up & $3.58 \mathrm{E}-17$ & $1.58 \mathrm{E}-15$ & calmodulin-like protein 5-like isoform 1 \\
\hline & Unigene13572_S2_1 & -1.44 & Down & $3.78 \mathrm{E}-18$ & 1.77E-16 & calmodulin-like protein 5-like \\
\hline & Unigene27042_S2_3 & 1.07 & Up & $1.59 \mathrm{E}-34$ & $1.46 \mathrm{E}-32$ & probable calcium-binding protein CML36-like \\
\hline & Unigene3462_S2_3 & -2.20 & Down & $6.33 \mathrm{E}-08$ & $1.30 \mathrm{E}-06$ & probable calcium-binding protein CML31 \\
\hline & Unigene5836_S2_1 & 2.50 & Up & $2.70 E-82$ & $6.16 \mathrm{E}-80$ & $\begin{array}{l}\text { CBL-interacting serine/threonine-protein } \\
\text { kinase 6-like isoform } 1\end{array}$ \\
\hline & CL2470.Contig1_S2_1 & 1.64 & Up & $2.74 \mathrm{E}-43$ & $3.21 \mathrm{E}-41$ & $\begin{array}{l}\text { CBL-interacting serine/threonine- } \\
\text { protein kinase 6-like isoform } 1\end{array}$ \\
\hline & Unigene82472_S2_1 & 1.52 & Up & $3.72 \mathrm{E}-06$ & $5.92 \mathrm{E}-05$ & $\begin{array}{l}\text { CBL-interacting serine/threonine- } \\
\text { protein kinase } 11\end{array}$ \\
\hline & Unigene71605_S2_1 & 1.36 & Up & $1.24 \mathrm{E}-54$ & $1.85 E-52$ & CBL-interacting protein kinase 18 \\
\hline & CL8813.Contig2_S2_1 & -3.32 & Down & $2.00 \mathrm{E}-29$ & $1.56 \mathrm{E}-27$ & $\begin{array}{l}\text { CBL-interacting serine/threonine- } \\
\text { protein kinase } 25 \text {-like }\end{array}$ \\
\hline & Unigene86355_S2_3 & 1.10 & Up & $2.01 \mathrm{E}-07$ & $3.84 \mathrm{E}-06$ & $\begin{array}{l}\text { calmodulin-binding receptor-like } \\
\text { cytoplasmic kinase } 3 \text {-like }\end{array}$ \\
\hline \multirow[t]{8}{*}{ CK vs C2 } & CL8580.Contig1_S2_3 & 13.00 & Up & $1.87 \mathrm{E}-27$ & $1.26 \mathrm{E}-25$ & cation/calcium exchanger 4-like \\
\hline & Unigene82641_S2_3 & 5.87 & Up & $1.18 \mathrm{E}-16$ & $4.68 \mathrm{E}-15$ & cation/calcium exchanger 4-like \\
\hline & Unigene21268_S2_3 & 5.47 & Up & $3.91 \mathrm{E}-24$ & $2.29 \mathrm{E}-22$ & cation/calcium exchanger 4 \\
\hline & Unigene13572_S2_1 & -2.00 & Down & $3.91 \mathrm{E}-27$ & $2.58 \mathrm{E}-25$ & calmodulin-like protein 5-like \\
\hline & Unigene74966_S2_1 & 1.38 & Up & $1.46 \mathrm{E}-13$ & 4.77E-12 & calmodulin-like protein 1-like \\
\hline & Unigene3462_S2_3 & -3.72 & Down & $2.81 \mathrm{E}-12$ & $8.28 \mathrm{E}-11$ & probable calcium-binding protein CML31 \\
\hline & Unigene7543_S2_3 & 2.17 & Up & $3.81 \mathrm{E}-08$ & 0.000000753 & probable calcium-binding protein CML10 \\
\hline & CL794.Contig2_S2_1 & 3.59 & Up & 0.00000641 & 0.0000922 & $\begin{array}{l}\text { CBL-interacting serine/threonine- } \\
\text { protein kinase }\end{array}$ \\
\hline
\end{tabular}


Table 2 The differential gene expression of genes involved in $\mathrm{Ca}^{2+}$ signalling pathway in each comparison (Continued)

\begin{tabular}{|c|c|c|c|c|c|c|}
\hline & Unigene5836_S2_1 & 2.44 & Up & $6.21 E-76$ & $1.23 \mathrm{E}-73$ & $\begin{array}{l}\text { CBL-interacting serine/threonine-protein } \\
\text { kinase 6-like isoform } 1\end{array}$ \\
\hline & CL4456.Contig1_S2_3 & 1.13 & Up & 8.1E-107 & $2.42 \mathrm{E}-104$ & $\begin{array}{l}\text { CBL-interacting serine/threonine-protein } \\
\text { kinase 6-like isoform } 1\end{array}$ \\
\hline & CL8813.Contig2_S2_1 & -3.00 & Down & 4.19E-26 & 2.67E-24 & $\begin{array}{l}\text { CBL-interacting serine/threonine-protein } \\
\text { kinase } 25 \text {-like }\end{array}$ \\
\hline & Unigene86355_S2_3 & 1.10 & Up & 0.000000279 & 0.00000491 & $\begin{array}{l}\text { calmodulin-binding receptor-like } \\
\text { cytoplasmic kinase } 3 \text {-like }\end{array}$ \\
\hline \multirow[t]{5}{*}{ CK vs B1 } & Unigene35781_S2_1 & 1.13 & Up & 3.2E-09 & 0.000000491 & sodium/calcium exchanger family protein \\
\hline & Unigene27711_S2_3 & 1.26 & Up & 4.74E-66 & 1.16E-62 & calcium-binding allergen Ole e 8-like \\
\hline & Unigene76667_S2_3 & 1.18 & Up & 4.71E-20 & $2 \mathrm{E}-17$ & calcium-binding protein CML24-like \\
\hline & Unigene26965_S2_3 & 1.06 & Up & $1.16 \mathrm{E}-10$ & $2.16 \mathrm{E}-08$ & probable calcium-binding protein CML48 \\
\hline & Unigene5836_S2_1 & 1.05 & Up & 8.75E-10 & 0.000000144 & $\begin{array}{l}\text { CBL-interacting serine/threonine-protein } \\
\text { kinase 6-like isoform } 1\end{array}$ \\
\hline \multirow[t]{9}{*}{ CK vs B2 } & Unigene35781_S2_1 & 1.15 & Up & $1.62 \mathrm{E}-09$ & 0.000000175 & sodium/calcium exchanger family protein \\
\hline & Unigene86214_S2_3 & 2.02 & Up & $1.08 \mathrm{E}-58$ & 1.17E-55 & calmodulin-like protein 5-like isoform 1 \\
\hline & Unigene13572_S2_1 & 1.21 & Up & 4.51E-30 & $1.89 \mathrm{E}-27$ & calmodulin-like protein 5-like \\
\hline & Unigene76667_S2_3 & 1.03 & Up & 4.6E-15 & $8.4 \mathrm{E}-13$ & calcium-binding protein CML24-like \\
\hline & Unigene27711_s2_3 & 1.66 & Up & $8.69 E-131$ & $2.78 \mathrm{E}-127$ & calcium-binding allergen Ole e 8-like \\
\hline & Unigene19284_S2_1 & 1.22 & Up & 0.000000074 & 0.00000616 & calcium-binding protein CAST-like \\
\hline & CL4878.Contig1_S2_1 & 1.05 & Up & 2.57E-19 & $6.24 \mathrm{E}-17$ & calcium-dependent protein kinase 9-like \\
\hline & Unigene5836_S2_1 & 2.08 & Up & $1.39 \mathrm{E}-48$ & $1.16 \mathrm{E}-45$ & $\begin{array}{l}\text { CBL-interacting serine/threonine-protein } \\
\text { kinase 6-like isoform } 1\end{array}$ \\
\hline & Unigene82472_S2_1 & 1.48 & Up & 0.000011 & 0.000600274 & $\begin{array}{l}\text { CBL-interacting serine/threonine- } \\
\text { protein kinase } 11\end{array}$ \\
\hline \multirow[t]{4}{*}{ A vs C1 } & Unigene27711_s2_3 & 1.70 & Up & $1.48 \mathrm{E}-57$ & $3.91 E-54$ & calcium-binding allergen Ole e 8-like \\
\hline & Unigene42277_S2_1 & 1.19 & Up & 0.000000676 & 0.0000976 & calcium-binding protein CML45-like \\
\hline & Unigene86214_S2_3 & 1.19 & Up & $1.4 \mathrm{E}-16$ & 7E-14 & calmodulin-like protein 5-like isoform 1 \\
\hline & CL2470.Contig1_S2_1 & 1.39 & Up & $2.71 \mathrm{E}-33$ & $3.4 \mathrm{E}-30$ & $\begin{array}{l}\mathrm{CBL} \text {-interacting serine/threonine-protein } \\
\text { kinase } 6 \text {-like isoform } 1\end{array}$ \\
\hline A vs C2 & Unigene27711_S2_3 & 1.45 & Up & $2.38 \mathrm{E}-38$ & $5.5 E-35$ & calcium-binding allergen Ole e 8-like \\
\hline
\end{tabular}

found in both, A vs $\mathrm{C} 1$ and A vs $\mathrm{C} 2$, while two HSPs were identified in the $\mathrm{A} v s \mathrm{C} 1$ comparison. The details of classes of protein of the CKA treatment are presented in Table 6.

\section{Verification of differential transcription using quantitative real time PCR (qPCR)}

To further verify the expression profiles of genes in our Illumina RNA-Seq results, we have performed a selection of 15 DTGs for their key roles in response to low temperature by qRT-PCR, these incorporated genes encoding serine/threonine-protein kinase, LEA protein, dehydrin, a gibberellin-regulated protein, a jasmonate ZIM-domain protein, and a DEAD-box ATP-dependent $\mathrm{RH}$, along with a selection of TFs (WRKY, DREB, AP2, bHLH and DOF). The qPCR outcomes in each case correlated closely with the transcript abundances estimated from the RNA-Seq output (Figure 3).

\section{Discussion}

\section{Global patterns of transcription in response to low} temperature

The information available on the molecular basis of the response of Chrysanthemum to low temperature is still meagre. However, the development of next-generation sequencing technology provides a straightforward method for the identification of genes involved in this process, and we can try to elaborate the molecular mechanism underlying the response to low temperature. Over $77 \%$ of the reads in each of the six RNA-Seq libraries corresponded with known transcripts (Table 1), a proportion which was as high as that achieved in a similar study of Anthurium [30]. The less than $23 \%$ of reads were unmapped probably as a result of unidentified transcripts [33]. Around 4,000 DTGs were identified in each of the CKA, CKC1 and CKC2 treatments (Figure 1). As was also the case for Camellia sinensis [28], the majority of DTGs involved uprather than down-regulation by the stress. In both species, 
Table 3 The differential gene expression of MAPK cascades genes in each comparison

\begin{tabular}{|c|c|c|c|c|c|c|}
\hline Comparison & GenelD & $\log 2$ Ratio & Up-down-regulation & P-value & FDR & Gene description \\
\hline \multirow[t]{2}{*}{ CK vs C1 } & Unigene36211_S2_1 & 1.90 & Up & $1.3 \mathrm{E}-10$ & 3.55E-09 & $\begin{array}{l}\text { mitogen-activated protein kinase kinase kinase } \\
\text { 3-like }\end{array}$ \\
\hline & Unigene36598_S2_1 & 1.04 & Up & 0.00000542 & 8.37E-05 & mitogen-activated protein kinase 16 \\
\hline CK vs C2 & Unigene36211_S2_1 & 1.93 & Up & 7.40E-11 & $1.94 \mathrm{E}-09$ & $\begin{array}{l}\text { mitogen-activated protein kinase kinase kinase } \\
\text { 3-like }\end{array}$ \\
\hline CK vs B1 & Unigene36211_S2_1 & 1.69 & Up & $5.43 \mathrm{E}-08$ & $6.92 \mathrm{E}-06$ & $\begin{array}{l}\text { mitogen-activated protein kinase kinase kinase } \\
\text { 3-like }\end{array}$ \\
\hline \multirow[t]{4}{*}{ CK vs B2 } & Unigene34028_S2_3 & 4.90 & Up & $2.29 \mathrm{E}-08$ & $2.06 \mathrm{E}-06$ & $\begin{array}{l}\text { mitogen-activated protein kinase kinase kinase } \\
\text { A-like }\end{array}$ \\
\hline & Unigene36211_S2_1 & 2.24 & Up & $1.32 \mathrm{E}-15$ & $2.52 \mathrm{E}-13$ & $\begin{array}{l}\text { mitogen-activated protein kinase kinase kinase } \\
\text { 3-like }\end{array}$ \\
\hline & Unigene8656_S2_1 & 2.12 & Up & $3.38 \mathrm{E}-07$ & $2.51 \mathrm{E}-05$ & $\begin{array}{l}\text { mitogen-activated protein kinase kinase kinase } \\
\text { ANP1-like }\end{array}$ \\
\hline & Unigene1412_S2_1 & 1.44 & Up & 3.32E-09 & $3.43 \mathrm{E}-07$ & $\begin{array}{l}\text { mitogen-activated protein kinase kinase kinase } \\
\text { ANP1-like }\end{array}$ \\
\hline A vs C1 & Unigene36211_S2_1 & 2.01 & Up & $5 E-11$ & 1.47E-08 & $\begin{array}{l}\text { mitogen-activated protein kinase kinase kinase } \\
\text { 3-like }\end{array}$ \\
\hline A vs C2 & Unigene36211_S2_1 & 2.03 & Up & $2.84 \mathrm{E}-11$ & 1.19E-08 & $\begin{array}{l}\text { mitogen-activated protein kinase kinase kinase } \\
\text { 3-like }\end{array}$ \\
\hline
\end{tabular}

many of the DTGs comprised genes associated with low temperature sensing or signal transduction, low temperature-responsive TFs, stabilization of the plasma membrane and osmosensing-responsiveness.

\section{Low temperature sensing and signaling genes}

Low temperature stress-induced signals are directed to various pathways [8]. $\mathrm{Ca}^{2+}$ is well recognized as a messenger in stress signalling [34], and is sensed by proteins of three main classes: CDPKs, CaMs and CBLs $[35,36]$. A further important pathway is the mitogen-activated protein kinases (MAPKs) cascade [8], while a number of receptor-like protein kinases (RLKs) are known to be responsible for perceiving changes in the external environment and transducing the appropriate signal $[37,38]$. The Arabidopsis and rice genomes harbor, respectively, 50 and 32 CML-encoding genes [39]. In rice, the transcription of the calmodulin-like OsMSR2 gene is significantly up-regulated by a series of stresses including low temperature in different tissues at different developmental stages, and its heterologous expression in A. thaliana has suggested that the gene affects salinity and drought tolerance in an ABA-dependent manner [40]. In Camellia sinensis exposed to low temperature, five calmodulin genes, two CDPK genes and one CBL gene were identified to be involved in signal transduction [28]. Here, five Unigenes resembling CML (Unigene74966_S2_1, 13572_ S2_1, 3462_S2_3, 76667_S2_3 and 42277_S2_1) were identified as significant DTGs in the CKA treatment, and three of these were also differentially transcribed in both the CKC1 and CKC2 treatments.
CBL-interacting protein kinases (CIPKs), which specifically interact with CBLs, are thought to act as sensors since they lack any enzymatic activity [41]. Three DTGs with homology to CIPK (Unigene5836_S2_1, CL4456.Contig1_S2_3 and CL8813.Contig2_S2_1) were identified in the CKA treatment. Both the CKC1 and CKC2 treatments also featured the same two DTGs (Unigene5836_S2_1 and CL8813.Contig2_S2_1), as well as the other three CIPK homologues (CL2470.Contig1_S2_1, Unigene82472_S2_1, Unigene71605_S2_1) in the former treatment, and two CIPK homologues (CL794.Contig2_S2_1, CL4456.Contig1_S2_3) in the latter. A number of CDPKs have been proven to participate in rapid abiotic stress and immune signaling responses [42]. Transgenic Arabidopsis heterologously expressing the Populus euphratica gene PeCPK10 show an enhanced level of freezing tolerance, perhaps through the transgene's enhancement of the transcript abundance of the abiotic stress-responsive genes $R D 29 B$ and COR15A [43]. Only one CDPK homologue (CL4878. Contig1_S2_1) was identified as a DTG in the CKB2 treatment, but no other homologues featured as DTGs in any of the other treatments. However, in A vs $\mathrm{C} 1$ comparison, with respect to $\mathrm{Ca}^{2+}$ signalling pathway, only two $C M L$ genes (Unigene42277_S2_1 and 86214_S2_3) and one CIPK gene (CL2470.Contig1_S2_1) were identified as DTGs. Four MAPKKK genes (Unigene34028_S2_3, Unigene36211_S2_1, Unigene8656_S2_1, and Unigene1412_S2_1) were differently transcribed in the CKB2 treatment. Unigene36211_S2_1 also featured in the CKB1 and $\mathrm{CKC} 2$ treatments, while a fifth $M A P K$ gene (Unigene-36598_S2_1) was found to transcribe differently in the CKC1 treatment. No one gene of MAPK cascade 
Table 4 The differential gene expression of Serine/threonine-protein kinase genes in each comparison

\begin{tabular}{|c|c|c|c|c|c|c|}
\hline Comparison & GenelD & $\begin{array}{l}\log 2 \\
\text { ratio }\end{array}$ & $\begin{array}{l}\text { Up-down- } \\
\text { regulation }\end{array}$ & P-value & FDR & Gene description \\
\hline \multirow[t]{28}{*}{ CK vs A } & Unigene48078_S2_3 & 4.56 & Up & 7.02E-79 & $1.54 \mathrm{E}-76$ & Serine/threonine-protein kinase Nek8 \\
\hline & CL6510.Contig1_S2_3 & 3.24 & Up & $1.54 \mathrm{E}-11$ & 4.57E-10 & serine/threonine-protein kinase SRK21-like \\
\hline & Unigene24393_S2_1 & 3.24 & Up & $8.16 \mathrm{E}-10$ & $2.05 \mathrm{E}-08$ & $\begin{array}{l}\text { probable serine/threonine-protein } \\
\text { kinase WNK11-like }\end{array}$ \\
\hline & Unigene84010_S2_3 & 2.88 & Up & 0.00000574 & 0.0000881 & $\begin{array}{l}\text { G-type lectin S-receptor-like serine/ } \\
\text { threonine-protein kinase RLK1-like }\end{array}$ \\
\hline & Unigene6145_S2_3 & 1.86 & Up & 0.00000809 & 0.000120748 & serine/threonine-protein kinase SRK2B-like \\
\hline & Unigene82172_S2_1 & 1.67 & Up & $5.09 \mathrm{E}-12$ & $1.58 \mathrm{E}-10$ & $\begin{array}{l}\text { LRR receptor-like serine/threonine- } \\
\text { protein kinase FLS2-like }\end{array}$ \\
\hline & CL5751.Contig4_S2_3 & 1.46 & Up & 4.35E-29 & $3.28 \mathrm{E}-27$ & serine/threonine-protein kinase GRIK2-like \\
\hline & Unigene77363_S2_3 & 1.08 & Up & $2.51 \mathrm{E}-14$ & $9.25 \mathrm{E}-13$ & $\begin{array}{l}\text { receptor-like serine/threonine-protein } \\
\text { kinase ALE2-like }\end{array}$ \\
\hline & CL2322.Contig2_S2_3 & 1.00 & Up & $2.16 \mathrm{E}-12$ & $6.91 \mathrm{E}-11$ & serine/threonine-protein kinase HT1-like \\
\hline & Unigene51530_S2_3 & -2.51 & Down & $7.52 \mathrm{E}-08$ & 0.00000153 & $\begin{array}{l}\text { LRR receptor-like serine/threonine- } \\
\text { protein kinase At } 4937250 \text {-like }\end{array}$ \\
\hline & CL2131.Contig1_S2_1 & -2.44 & Down & $1.66 \mathrm{E}-57$ & $2.62 \mathrm{E}-55$ & $\begin{array}{l}\text { G-type lectin S-receptor-like } \\
\text { serine/threonine-protein kinase RLK1-like }\end{array}$ \\
\hline & Unigene74903_S2_1 & -2.21 & Down & 1.68E-14 & $6.25 \mathrm{E}-13$ & $\begin{array}{l}\text { LRR receptor-like serine/threonine- } \\
\text { protein kinase At4g37250-like }\end{array}$ \\
\hline & CL102.Contig1_S2_1 & -2.04 & Down & 0.0000201 & 0.000280649 & serine/threonine-protein kinase At5g41260 \\
\hline & Unigene86891_S2_3 & -1.76 & Down & 0.000000168 & 0.00000325 & $\begin{array}{l}\text { LRR receptor-like serine/threonine- } \\
\text { protein kinase At1g53430 }\end{array}$ \\
\hline & Unigene62396_S2_1 & -1.75 & Down & $1.86 \mathrm{E}-17$ & $8.16 \mathrm{E}-16$ & $\begin{array}{l}\text { LRR receptor-like serine/threonine- } \\
\text { protein kinase At1g07650-like }\end{array}$ \\
\hline & Unigene5608_S2_1 & -1.71 & Down & $2.73 E-22$ & $1.55 \mathrm{E}-20$ & $\begin{array}{l}\text { LRR receptor-like serine/threonine- } \\
\text { protein kinase At1g56130-like isoform } 1\end{array}$ \\
\hline & Unigene82743_S2_1 & -1.51 & Down & 0.000000303 & 0.00000565 & $\begin{array}{l}\text { LRR receptor-like serine/threonine- } \\
\text { protein kinase At } 4937250 \text {-like }\end{array}$ \\
\hline & CL6249.Contig1_S2_1 & -1.15 & Down & $1.28 \mathrm{E}-10$ & $3.51 \mathrm{E}-09$ & $\begin{array}{l}\text { receptor-like serine/threonine-protein } \\
\text { kinase At2g45590-like }\end{array}$ \\
\hline & Unigene599_S2_1 & 1.94 & Up & $5.48 \mathrm{E}-16$ & $1.08 \mathrm{E}-13$ & $\begin{array}{l}\text { LRR receptor-like serine/threonine- } \\
\text { protein kinase At3g47570-like }\end{array}$ \\
\hline & CL528.Contig2_S2_1 & 1.85 & Up & 0.0000101 & 0.000555903 & $\begin{array}{l}\text { G-type lectin S-receptor-like serine/ } \\
\text { threonine-protein kinase SD2-5-like }\end{array}$ \\
\hline & CL11839.Contig2_S2_3 & 1.56 & Up & 0.000000918 & 0.0000626 & $\begin{array}{l}\text { G-type lectin S-receptor-like serine/ } \\
\text { threonine-protein kinase At1g34300-like }\end{array}$ \\
\hline & CL11232.Contig1_S2_3 & 1.46 & Up & 1.35E-11 & 1.85E-09 & serine/threonine-protein kinase RLCKVII-like \\
\hline & Unigene7157_S2_3 & 1.45 & Up & 7.74E-08 & 0.00000642 & $\begin{array}{l}\text { LRR receptor-like serine/threonine- } \\
\text { protein kinase GSO1-like }\end{array}$ \\
\hline & Unigene82172_S2_1 & 1.38 & Up & 6.47E-08 & 0.00000544 & $\begin{array}{l}\text { LRR receptor-like serine/threonine-protein } \\
\text { kinase FLS2-like }\end{array}$ \\
\hline & CL5137.Contig1_S2_1 & 1.27 & Up & $2.98 \mathrm{E}-09$ & 0.00000031 & $\begin{array}{l}\text { LRR receptor-like serine/threonine-protein } \\
\text { kinase GSO1-like }\end{array}$ \\
\hline & Unigene35893_S2_1 & 1.09 & Up & $1.1 \mathrm{E}-09$ & 0.000000122 & $\begin{array}{l}\text { G-type lectin S-receptor-like serine/ } \\
\text { threonine-protein kinase SD2-5-like }\end{array}$ \\
\hline & Unigene13659_S2_3 & 1.01 & Up & 0.00000506 & 0.000296619 & $\begin{array}{l}\text { inactive leucine-rich repeat receptor-like } \\
\text { serine/threonine-protein kinase }\end{array}$ \\
\hline & Unigene62396_S2_1 & -1.43 & Down & 2.37E-13 & $3.75 E-11$ & $\begin{array}{l}\text { LRR receptor-like serine/threonine- } \\
\text { protein kinase At1g07650-like }\end{array}$ \\
\hline \multirow[t]{2}{*}{ CK vs C1 } & Unigene48078_S2_3 & 4.86 & Up & $1.6412 \mathrm{E}-101$ & 4.5942E-99 & Serine/threonine-protein kinase Nek8, putative \\
\hline & Unigene24393_S2_1 & 3.17 & Up & 2.28788E-09 & 5.51317E-08 & serine/threonine-protein kinase WNK11-like \\
\hline
\end{tabular}


Table 4 The differential gene expression of Serine/threonine-protein kinase genes in each comparison (Continued)

\begin{tabular}{|c|c|c|c|c|c|c|}
\hline & CL6510.Contig1_S2_3 & 2.74 & Up & $1.7455 \mathrm{E}-07$ & 3.36939E-06 & serine/threonine-protein kinase SRK2l-like \\
\hline & Unigene6145_S2_3 & 2.13 & Up & $6.15014 \mathrm{E}-08$ & 1.26115E-06 & serine/threonine-protein kinase SRK2B-like \\
\hline & Unigene77360_\$2_3 & 1.83 & Up & $5.63286 \mathrm{E}-05$ & 0.000724311 & $\begin{array}{l}\text { probable serine/threonine-protein kinase } \\
\text { At1g54610-like }\end{array}$ \\
\hline & CL8653.Contig4_S2_3 & 1.72 & Up & 2.68074E-05 & 0.000365143 & serine/threonine-protein kinase HT1-like \\
\hline & CL812.Contig8_S2_3 & 1.65 & Up & $6.44362 \mathrm{E}-05$ & 0.000817344 & serine/threonine-protein kinase SAPK3 \\
\hline & CL2322.Contig2_S2_3 & 1.33 & Up & $1.25092 \mathrm{E}-23$ & 7.73232E-22 & serine/threonine-protein kinase HT1-like \\
\hline & CL5751.Contig4_S2_3 & 1.33 & Up & $5.47232 \mathrm{E}-24$ & $3.44379 \mathrm{E}-22$ & serine/threonine-protein kinase GRIK2-like \\
\hline & CL3545.Contig5_S2_3 & 1.19 & Up & $2.89914 \mathrm{E}-31$ & 2.39887E-29 & serine/threonine-protein kinase At5g41260 \\
\hline & Unigene82172_S2_1 & 1.09 & Up & 0.000041836 & 0.000548576 & $\begin{array}{l}\text { LRR receptor-like serine/threonine- } \\
\text { protein kinase FLS2-like }\end{array}$ \\
\hline & Unigene42172_S2_3 & 1.02 & Up & $2.83884 \mathrm{E}-23$ & 1.71985E-21 & $\begin{array}{l}\text { serine/threonine-protein kinase } \\
\text { AtPK2/AtPK19-like }\end{array}$ \\
\hline & Unigene85255_S2_3 & 1.01 & Up & 1.19271E-05 & 0.000173177 & $\begin{array}{l}\text { receptor-like serine/threonine- } \\
\text { protein kinase At2g45590-like }\end{array}$ \\
\hline & Unigene51530_S2_3 & -3.58 & Down & $7.18962 \mathrm{E}-11$ & $1.99504 E-09$ & $\begin{array}{l}\text { LRR receptor-like serine/threonine- } \\
\text { protein kinase At4g37250-like }\end{array}$ \\
\hline & Unigene74903_S2_1 & -2.56 & Down & 1.88547E-17 & $8.4618 \mathrm{E}-16$ & $\begin{array}{l}\text { LRR receptor-like serine/threonine- } \\
\text { protein kinase At4g37250-like }\end{array}$ \\
\hline & CL102.Contig1_S2_1 & -2.28 & Down & $3.3508 \mathrm{E}-06$ & 5.3771E-05 & serine/threonine-protein kinase At5g41260 \\
\hline & Unigene74228_S2_1 & -2.12 & Down & $2.61668 \mathrm{E}-63$ & 4.61793E-61 & serine/threonine-protein kinase cx32, putative \\
\hline & CL2131.Contig1_S2_1 & -1.93 & Down & $1.20746 \mathrm{E}-44$ & $1.451 \mathrm{E}-42$ & $\begin{array}{l}\text { G-type lectin S-receptor-like serine/ } \\
\text { threonine-protein kinase RLK1-like }\end{array}$ \\
\hline & Unigene86891_S2_3 & -1.90 & Down & $2.01214 \mathrm{E}-08$ & 4.3637E-07 & $\begin{array}{l}\text { LRR receptor-like serine/threonine- } \\
\text { protein kinase At1g53430 }\end{array}$ \\
\hline & CL6249.Contig1_S2_1 & -1.45 & Down & $8.25782 \mathrm{E}-15$ & $3.1245 \mathrm{E}-13$ & $\begin{array}{l}\text { receptor-like serine/threonine-protein kinase } \\
\text { At2g45590-like }\end{array}$ \\
\hline & Unigene5608_S2_1 & -1.39 & Down & $2.98124 \mathrm{E}-17$ & 1.32493E-15 & $\begin{array}{l}\text { LRR receptor-like serine/threonine- } \\
\text { protein kinase At1g56130-like isoform } 1\end{array}$ \\
\hline & Unigene28326_S2_1 & -1.11 & Down & $1.13529 E-22$ & $6.61992 \mathrm{E}-21$ & $\begin{array}{l}\text { receptor-like serine/threonine-protein } \\
\text { kinase ALE2-like }\end{array}$ \\
\hline \multirow[t]{13}{*}{ CK vs C2 } & CL10252.Contig2_S2_1 & 10.43 & Up & 0.0000214 & 0.000279936 & serine/threonine-protein kinase HT1-like \\
\hline & Unigene48078_S2_3 & 4.65 & Up & $1.32 \mathrm{E}-84$ & $2.96 \mathrm{E}-82$ & Serine/threonine-protein kinase Nek8, putative \\
\hline & CL6510.Contig1_S2_3 & 3.48 & Up & $3.57 \mathrm{E}-14$ & $1.21 \mathrm{E}-12$ & serine/threonine-protein kinase SRK2I-like \\
\hline & Unigene24393_S2_1 & 2.79 & Up & 0.00000126 & 0.0000204 & probable serine/threonine-protein kinase WNK11-like \\
\hline & Unigene6145_S2_3 & 2.32 & Up & $1.64 \mathrm{E}-09$ & $3.76 \mathrm{E}-08$ & serine/threonine-protein kinase SRK2B-like \\
\hline & CL8653.Contig4_S2_3 & 2.21 & Up & 4.68E-09 & 0.000000102 & serine/threonine-protein kinase HT1-like \\
\hline & Unigene82172_S2_1 & 1.81 & Up & $1.26 \mathrm{E}-14$ & $4.39 \mathrm{E}-13$ & $\begin{array}{l}\text { LRR receptor-like serine/threonine-protein } \\
\text { kinase FLS2-like }\end{array}$ \\
\hline & CL5751.Contig4_S2_3 & 1.42 & Up & $2.22 \mathrm{E}-27$ & $1.48 \mathrm{E}-25$ & serine/threonine-protein kinase GRIK2-like \\
\hline & Unigene98225_S2_3 & 1.32 & Up & 0.0000032 & 0.0000484 & $\begin{array}{l}\text { probable LRR receptor-like serine/threonine-protein } \\
\text { kinase At2g24230-like }\end{array}$ \\
\hline & CL1564.Contig1_S2_1 & 1.15 & Up & $1.15 \mathrm{E}-08$ & 0.000000243 & serine/threonine-protein kinase AtPK2/AtPK19-like \\
\hline & CL3545.Contig5_S2_3 & 1.01 & Up & $3.54 \mathrm{E}-21$ & $1.8 \mathrm{E}-19$ & probable serine/threonine-protein kinase At5g41260 \\
\hline & CL2492.Contig1_S2_1 & -10.17 & Down & 0.0000845 & 0.000986827 & $\begin{array}{l}\text { G-type lectin S-receptor-like serine/threonine-protein } \\
\text { kinase At4g27290-like }\end{array}$ \\
\hline & Unigene51530_\$2_3 & -2.71 & Down & $1.86 \mathrm{E}-08$ & 0.000000383 & $\begin{array}{l}\text { probable LRR receptor-like serine/threonine-protein } \\
\text { kinase At4g37250-like }\end{array}$ \\
\hline
\end{tabular}


Table 4 The differential gene expression of Serine/threonine-protein kinase genes in each comparison (Continued)

\begin{tabular}{|c|c|c|c|c|c|}
\hline CL2131.Contig1_S2_1 & -2.20 & Down & $3.39 \mathrm{E}-51$ & $4.56 \mathrm{E}-49$ & $\begin{array}{l}\text { G-type lectin S-receptor-like serine/threonine-protein } \\
\text { kinase RLK1-like }\end{array}$ \\
\hline Unigene74499_S2_1 & -2.07 & Down & 0.0000471 & 0.000577174 & $\begin{array}{l}\text { probable leucine-rich repeat receptor-like serine/ } \\
\text { threonine-protein kinase At5g15730 }\end{array}$ \\
\hline CL102.Contig1_S2_1 & -2.05 & Down & 0.0000183 & 0.000241927 & probable serine/threonine-protein kinase At5g41260 \\
\hline Unigene62396_S2_1 & -2.02 & Down & $4.95 \mathrm{E}-21$ & $2.49 \mathrm{E}-19$ & $\begin{array}{l}\text { LRR receptor-like serine/threonine-protein kinase } \\
\text { At1g07650-like }\end{array}$ \\
\hline Unigene74903_S2_1 & -1.89 & Down & $4.06 \mathrm{E}-12$ & $1.18 \mathrm{E}-10$ & $\begin{array}{l}\text { LRR receptor-like serine/threonine-protein kinase } \\
\text { At4g37250-like }\end{array}$ \\
\hline Unigene5608_S2_1 & -1.76 & Down & $3.3 \mathrm{E}-23$ & $1.86 \mathrm{E}-21$ & $\begin{array}{l}\text { LRR receptor-like serine/threonine-protein kinase } \\
\text { At1g56130-like isoform } 1\end{array}$ \\
\hline Unigene86891_S2_3 & -1.33 & Down & 0.0000185 & 0.000245014 & $\begin{array}{l}\text { probable LRR receptor-like serine/threonine-protein } \\
\text { kinase At1g53430 }\end{array}$ \\
\hline Unigene13930_S2_1 & -1.22 & Down & $1.29 \mathrm{E}-13$ & $4.23 \mathrm{E}-12$ & $\begin{array}{l}\text { LRR receptor-like serine/threonine-protein } \\
\text { kinase GSO1 }\end{array}$ \\
\hline
\end{tabular}

was identified in the CKA treatment. In addition, many DTGs encoding serine/threonine-protein kinases were found in the CKA, CKC1 and CKC2 treatments; however, no gene was detected in the comparisons, A vs $\mathrm{C} 1$ and $\mathrm{A} v s \mathrm{C} 2$. These findings provided evidence for the crucial role of $\mathrm{Ca}^{2+}$ in the low temperature acclimation process in $C$. nankingense, and further proved that the MAPK pathway and serine/threonine-protein kinases are more strongly involved in the response to freezing.

\section{Major classes of TF involved in the response to low temperature}

Transcriptional regulation of stress-responsive genes is a vital component of the response to both abiotic and biotic stress [44]. Five major TF classes (AP2/ERF, bHLH, WRKY, TCP and MYB) were identified as DTGs in the treatments involved in a process of low temperature acclimation. The AP2/ERF family, which a large group of plant-specific transcription factors, has been sub-divided into AP2, RAV, ERF and DREB TFs [45]. DREBs control the ABA-independent transcription of low temperature responsive genes in A. thaliana [10]; the AtDREB1 subfamily harbors six members [46], of which $D R E B 1 A$ / $C B F 3, D R E B 1 B / C B F 1$ and $D R E B 1 C / C B F 2$ are the ones which respond most rapidly to low temperature. $A$. thaliana plants constitutively expressing any one of these TFs display a heightened tolerance to freezing, drought and salinity [45]. The DREB2B TF present in the desertadapted plant Eremosparton songoricum has been shown to enhance the tolerance of both yeast and tobacco against a variety of abiotic stresses. The constitutive expression in tobacco of EsDREB2B promotes the accumulation of proline in response to abiotic stress (including low temperature) [47]. Here, the DREB2 homologue
Unigene73473_S2_1 was up-regulated upon exposure to low temperature acclimation. It was speculated that the DREB2 may be involved in the accumulation of proline in response to low temperature. It is well known Proline accumulates in many plant species in response to environmental stress [48]. The constitutive expression of an AP2 TF has been shown to improve the tolerance of $A$. thaliana to low temperature, as well as to drought and high temperature [49]. Here, three AP2like genes (Unigene27271_S2_3, Unigene27661_S2_3 and CL1514.Contig4_S2_1) were also all up-regulated by CA. A number of other TFs, belonging to the WRKY, bHLH, TCP, MYB, MYC, Trihelix and b-ZIP families were also among the DTGs identified in treatments involving low temperature acclimation. The class of b-ZIP transcription factors, ABRE binding proteins (AREBs or ABFs), can bind to ABRE and activate ABA-dependent gene expression when plants are exposed to low temperature [10]. Many researches have also indicated that three families of transcription factors: WRKY, bHLH and MYB closely related to plant cold stress [50-52]. Five of six WRKYs, which were detected as DTGs, were downregulated in CK vs A comparison. However, three WRKYs were all up-regulated in A vs C1, and no WRKY as DTG was found in A vs $\mathrm{C} 2$. These findings suggested that WRKY family played essential and different roles in CA and early freezing response. Most ERFs also showed down-regulated in CK vs A comparison, while, one ERF was detected up-regulated as DTG in A vs C1; on extending the freezing treatment, five ERFs as DTGs were all up-regulated in A vs C2. The ERFs performing under different temperature treatment showed its roles in CA and cold tolerance. Few reports to date have indicated that TCP transcription factors involve in the response to cold stress. 
Table 5 The differential gene expression of Transcription factors (TFs) in each comparison

\begin{tabular}{|c|c|c|c|c|c|c|}
\hline Comparison & GenelD & $\log 2$ ratio & Up-down-regulation & P-value & FDR & Gene description \\
\hline \multirow[t]{41}{*}{ CK vs A } & CL695.Contig1_S2_3 & 3.21 & Up & $1.51 \mathrm{E}-09$ & $3.71 \mathrm{E}-08$ & WRKY transcription factor 4 \\
\hline & Unigene75748_S2_1 & -3.51 & Down & $2.15 \mathrm{E}-10$ & 5.76E-09 & WRKY transcription factor 1 \\
\hline & CL9703.Contig1_S2_1 & -3.16 & Down & 2.7E-14 & $9.92 \mathrm{E}-13$ & WRKY transcription factor 1 \\
\hline & Unigene28584_S2_1 & -1.56 & Down & 7.46E-80 & $1.66 \mathrm{E}-77$ & WRKY transcription factor \\
\hline & CL4806.Contig1_S2_1 & -1.51 & Down & 2.17E-06 & 0.0000358 & WRKY domain class transcription factor \\
\hline & Unigene80749_S2_1 & -1.37 & Down & 0.000019 & 0.000266621 & WRKY transcription factor 7-like \\
\hline & Unigene93511_S2_3 & 5.77 & Up & $1.63 \mathrm{E}-15$ & $6.48 \mathrm{E}-14$ & Ethylene-responsive transcription factor \\
\hline & Unigene79121_S2_1 & -10.32 & Down & 0.0000119 & 0.000171947 & ethylene-responsive transcription factor-like \\
\hline & CL3891.Contig1_S2_1 & -1.35 & Down & $8.09 \mathrm{E}-37$ & $7.84 \mathrm{E}-35$ & ethylene-responsive transcription factor 5 \\
\hline & Unigene25018_S2_1 & -2.85 & Down & $2 \mathrm{E}-34$ & $1.81 \mathrm{E}-32$ & ethylene-responsive transcription factor 5 \\
\hline & Unigene36230_S2_1 & -2.58 & Down & $3.31 \mathrm{E}-32$ & $2.78 \mathrm{E}-30$ & Ethylene-responsive transcription factor \\
\hline & Unigene73751_S2_1 & -2.38 & Down & $2.45 \mathrm{E}-22$ & $1.4 \mathrm{E}-20$ & $\begin{array}{l}\text { ethylene-responsive transcription factor } \\
\text { RAP2-4-like }\end{array}$ \\
\hline & Unigene83309_S2_1 & 3.07 & Up & 0.0000156 & 0.000221008 & DREBa \\
\hline & Unigene73473_S2_1 & 2.33 & Up & $9.16 \mathrm{E}-69$ & $1.73 \mathrm{E}-66$ & DREB2 transcription factor \\
\hline & Unigene27271_S2_3 & 1.39 & Up & $1.45 \mathrm{E}-13$ & $5.04 \mathrm{E}-12$ & AP2 transcription factor \\
\hline & Unigene27661_S2_3 & 1.38 & Up & $1.74 \mathrm{E}-57$ & $2.73 \mathrm{E}-55$ & AP2 domain class transcription factor \\
\hline & CL1514.Contig4_S2_1 & 1.30 & Up & $9.54 \mathrm{E}-60$ & $1.55 \mathrm{E}-57$ & AP2 domain class transcription factor \\
\hline & Unigene85419_S2_3 & -1.85 & Down & $1.18 \mathrm{E}-07$ & 0.00000234 & AP2/EREBP transcription factor ERF-2 \\
\hline & Unigene27190_S2_3 & 1.39 & Up & 2.77E-72 & 5.57E-70 & TCP family transcription factor TCP4 \\
\hline & Unigene14147_S2_3 & 1.08 & Up & $6.58 \mathrm{E}-41$ & 7.14E-39 & TB1-like TCP family transcription factor \\
\hline & CL1091.Contig3_S2_1 & -1.27 & Down & 3.63E-06 & 0.0000576 & TCP domain class transcription factor \\
\hline & Unigene97493_S2_3 & -2.25 & Down & 0.0000771 & 0.000963215 & transcription factor bHLH47-like \\
\hline & CL8515.Contig3_S2_1 & -1.04 & Down & 5.01E-06 & 0.0000776 & transcription factor bHLH13 \\
\hline & Unigene37079_S2_1 & 2.45 & Up & $1.16 \mathrm{E}-25$ & $7.68 \mathrm{E}-24$ & transcription factor bHLH128-like \\
\hline & Unigene28502_S2_1 & 1.58 & Up & $3.48 \mathrm{E}-47$ & 4.44E-45 & transcription factor bHLH130-like \\
\hline & Unigene28413_S2_1 & -1.67 & Down & $8.22 \mathrm{E}-24$ & $5 E-22$ & transcription factor MYC2-like \\
\hline & CL2771.Contig2_S2_3 & -1.66 & Down & 8.56E-08 & 0.00000172 & MYC1b transcription factor \\
\hline & Unigene36464_S2_1 & -1.37 & Down & $1.43 \mathrm{E}-07$ & 0.0000028 & transcription factor MYB44-like \\
\hline & Unigene56969_S2_3 & 2.92 & Up & $2.06 \mathrm{E}-62$ & $3.51 \mathrm{E}-60$ & DOF domain class transcription factor \\
\hline & Unigene13328_S2_3 & 1.77 & Up & 2.7E-105 & 7.79E-103 & trihelix transcription factor GTL2-like \\
\hline & Unigene84739_S2_3 & 1.06 & Up & $2.41 \mathrm{E}-07$ & 0.00000458 & bZIP transcription factor 60-like \\
\hline & CL12660.Contig2_S2_3 & 2.17 & Up & $2.66 \mathrm{E}-23$ & $1.59 \mathrm{E}-21$ & transcription factor GTE1-like \\
\hline & Unigene43245_S2_1 & 2.16 & Up & 0.0000208 & 0.00028917 & transcription factor VIP1-like \\
\hline & CL9834.Contig2_S2_1 & 1.69 & Up & $3.6 \mathrm{E}-10$ & 9.42E-09 & global transcription factor group \\
\hline & CL3990.Contig3_S2_1 & 1.25 & Up & $4.42 \mathrm{E}-55$ & $6.62 \mathrm{E}-53$ & transcription factor BTF3 \\
\hline & Unigene27985_S2_1 & 1.23 & Up & 3.7E-11 & $1.06 \mathrm{E}-09$ & transcription factor DIVARICATA-like \\
\hline & Unigene76924_S2_3 & 1.18 & Up & $3 \mathrm{E}-17$ & $1.31 \mathrm{E}-15$ & transcription factor BIM1-like \\
\hline & Unigene62871_S2_1 & -5.49 & Down & 4.63E-49 & $6.09 \mathrm{E}-47$ & transcription factor HEC1 \\
\hline & CL6640.Contig2_S2_1 & -1.70 & Down & 7.15E-44 & $8.36 \mathrm{E}-42$ & transcription factor, putative \\
\hline & CL5647.Contig3_S2_3 & -1.64 & Down & 0.0000377 & 0.000500576 & nuclear transcription factor $Y$ \\
\hline & CL7343.Contig2_S2_1 & -1.14 & Down & $5.47 \mathrm{E}-09$ & 0.000000126 & transcription factor RF2b \\
\hline \multirow[t]{2}{*}{ A vs C1 } & CL4444.Contig1_S2_1 & 10.93 & Up & 0.0000031 & 0.000374499 & $\begin{array}{l}\text { dehydration-responsive element-binding } \\
\text { factor } 1\end{array}$ \\
\hline & Unigene25410_S2_1 & 4.68 & Up & $2.16 \mathrm{E}-07$ & 0.0000352 & WRKY transcription factor 1 \\
\hline
\end{tabular}


Table 5 The differential gene expression of Transcription factors (TFs) in each comparison (Continued)

\begin{tabular}{|c|c|c|c|c|c|c|}
\hline & Unigene75748_S2_1 & 3.54 & Up & $1.17 \mathrm{E}-10$ & $3.25 \mathrm{E}-08$ & WRKY transcription factor 1 \\
\hline & CL9703.Contig1_S2_1 & 2.91 & Up & $2.26 \mathrm{E}-11$ & 6.85E-09 & WRKY transcription factor 1 \\
\hline & Unigene85931_S2_3 & 1.91 & Up & 7.27E-29 & 7.63E-26 & ethylene response factor 7 \\
\hline \multirow[t]{7}{*}{ A vs C2 } & CL4444.Contig1_S2_1 & 12.92 & Up & 2.65E-22 & 2.88E-19 & $\begin{array}{l}\text { dehydration-responsive } \\
\text { element-binding factor } 1\end{array}$ \\
\hline & Unigene5394_S2_1 & 11.27 & Up & $8.36 \mathrm{E}-21$ & $8.42 \mathrm{E}-18$ & $\begin{array}{l}\text { ethylene-responsive transcription } \\
\text { factor ERF109-like }\end{array}$ \\
\hline & Unigene47799_S2_3 & 11.06 & Up & $1.28 \mathrm{E}-07$ & 0.0000303 & $\begin{array}{l}\text { ethylene-responsive transcription } \\
\text { factor ERF017 }\end{array}$ \\
\hline & Unigene97493_S2_3 & 3.83 & Up & $2.12 \mathrm{E}-19$ & $1.81 \mathrm{E}-16$ & transcription factor bHLH47-like isoform 1 \\
\hline & Unigene13303_S2_3 & 1.89 & Up & $3.19 \mathrm{E}-32$ & $6.01 E-29$ & Ethylene-responsive transcription factor \\
\hline & Unigene25018_S2_1 & 1.52 & Up & $2.98 \mathrm{E}-07$ & 0.0000653 & ethylene-responsive transcription factor 5 \\
\hline & Unigene85931_S2_3 & 1.27 & Up & $4.38 \mathrm{E}-11$ & 0.000000018 & ethylene response factor 7 \\
\hline
\end{tabular}

Low temperature responsive genes related to post-transcriptional regulation

Post-transcriptional regulation (pre-mRNA processing, mRNA stabilization and mRNA export from the nucleus) has been implicated in the process of low temperature acclimation [7]. DEAD-box RHs are intimately associated with RNA-mediated processes and are related to various RNA metabolism events, including RNA synthesis to RNA degradation by means of catalyzing the ATP-dependent unwinding of local RNA secondary structures [53]. The transcription of the genes encoding these proteins is known to be regulated by stress in both bacteria and plants [54-57]. In A. thaliana, RH25 has been associated with enhanced freezing tolerance, probably through its function as an RNA chaperone [58]. The product of $R C F 1$, a low temperature-inducible $R H$ gene, is important for low temperature-responsive gene regulation and low temperature tolerance in plants through maintenance of normal pre-mRNA splicing instead of regulating mRNA export like a previously reported DEAD-box RH (LOS4) regulates mRNA export [59]. It has been demonstrated that the functional roles and RNA chaperone activity related to intron splicing in mitochondrial and chloroplast [59-62]. Here, respectively, eleven, nine and twelve genes encoding DEAD-box RHs were up-regulated in the CKA, $\mathrm{CKC1}$ and $\mathrm{CKC} 2$ treatments, but none were differentially transcribed in either the CKB1 or the CKB2 treatment; this was taken to imply that DEAD-box RHs are activated during the process of low temperature acclimation. In future, a major task is to clear how RNA chaperones recognize substrate RNAs and how they work with other proteins to regulate post-transcriptional RNA metabolism in response to developmental and environmental condition [53].

\section{Genes encoding functional proteins}

Besides protein factors involved in further regulation of signal transduction and gene expression such as transcription factor and protein kinase that probably function in stress response, various functional proteins featured as products of the DTGs, in particular, LEA protein and dehydrin, LEA proteins are accumulated during the late stage of seed maturation and under moisture deficient conditions, and act to protect higher plants from damage caused by abiotic stress. When the maize gene $Z m L E A 3$ is expressed in tobacco and yeast, it improves the plant's level of tolerance against both osmotic and oxidative stress [63]. The wheat LEA protein gene WCI16 expressed heterologously in A. thaliana enhances freezing tolerance [64]. Here, seven LEA protein genes were among the DTGs identified in the CKA treatment, five in both the CKC1 and $\mathrm{CKC} 2$ treatment, none were present in either the CKB1 or the CKB2 treatment, and only one was identified in both comparisons of $\mathrm{A} v s \mathrm{C} 1$ and $\mathrm{A} v s \mathrm{C} 2$. The implication was that LEA proteins probably enhance low temperature tolerance through their participation in the low temperature acclimation process. Dehydrins constitute a group of plant proteins involved in tolerance to low temperature and drought [65]. The thranscrips of genes encoding three dehydrins in E. globulus accumulate strongly in the stem and leaf tissue of acclimated plants, compared to non-acclimated [66]. Here, four genes encoding dehydrins were identified as DTGs in the CKA, CKC1 and $\mathrm{CKC} 2$ treatments. But none in either the CKB1 or CKB2 treatments, suggesting that, as with the LEA protein genes, their contribution to low temperature tolerance is expressed during the low temperature acclimation process. HSPs were initially identified from their involvement in the response to high temperatures, but it is now recognized that many of them also respond to low temperature. It is well known HSPs can protect plants against stress by means of reestablishing normal protein conformation and thus cellular homeostasis. Five major HSP families have been conservely defined, based on their molecular weight: HSP100s, HSP90s, HSP70s, HSP60s and small HSPs. Five 
Table 6 The differential gene expression of genes encoding LEA protein, HSPs, and RNA helicase in treatment CKA

\begin{tabular}{|c|c|c|c|c|c|c|}
\hline Related genes & GenelD & $\log 2$ ratio & Up-down-regulation & P-value & FDR & Gene description \\
\hline \multirow[t]{8}{*}{ LEA } & CL1591.Contig3_S2_3 & 11.63 & Up & 0.00000056 & 0.0000101 & $\begin{array}{l}\text { late embryogenesis abundant } \\
\text { protein-like protein }\end{array}$ \\
\hline & Unigene13900_S2_3 & 8.57 & Up & 0 & 0 & $\begin{array}{l}\text { late embryogenesis } \\
\text { abundant protein } 1\end{array}$ \\
\hline & CL3193.Contig2_S2_3 & 6.39 & Up & 4.99E-161 & $2.16 \mathrm{E}-158$ & $\begin{array}{l}\text { Late embryogenesis } \\
\text { abundant protein }\end{array}$ \\
\hline & CL11733.Contig3_S2_3 & 10.83 & Up & 0 & 0 & LEA1 protein \\
\hline & CL11733.Contig1_S2_3 & 8.27 & Up & 2.69098E-90 & 6.61827E-88 & LEA1 protein \\
\hline & Unigene28071_S2_1 & 4.42 & Up & 0 & 0 & LEA5 \\
\hline & CL3193.Contig2_S2_3 & 6.39 & Up & 4.9929E-161 & $2.158 \mathrm{E}-158$ & $\begin{array}{l}\text { Late embryogenesis } \\
\text { abundant protein Dc3 }\end{array}$ \\
\hline & Unigene14063_S2_1 & -1.16 & Down & 0.00000526 & 0.0000813 & $\begin{array}{l}\text { late embryogenesis } \\
\text { abundant protein } 3 \mathrm{~L}-1\end{array}$ \\
\hline \multirow[t]{6}{*}{ HSPS } & CL337.Contig29_S2_3 & 2.81 & Up & $3.39 \mathrm{E}-11$ & $9.79 \mathrm{E}-10$ & $\begin{array}{l}\text { putative heat shock } \\
\text { protein } 90 \text { family protein }\end{array}$ \\
\hline & CL1609.Contig2_S2_1 & 2.29 & Up & $8.01 \mathrm{E}-18$ & $3.62 \mathrm{E}-16$ & heat shock protein 90 \\
\hline & CL1609.Contig12_S2_1 & 1.67 & Up & $1.73 \mathrm{E}-10$ & 4.67E-09 & heat shock protein 90-2 \\
\hline & CL1609.Contig19_S2_1 & 1.30 & Up & $2.44 \mathrm{E}-16$ & $1.02 \mathrm{E}-14$ & heat shock protein 90 \\
\hline & CL6923.Contig2_S2_3 & 1.26 & Up & $3.84 \mathrm{E}-71$ & $7.6 \mathrm{E}-69$ & heat shock protein, putative \\
\hline & CL1609.Contig10_S2_1 & 1.08 & Up & $5.81 \mathrm{E}-27$ & $4.02 E-25$ & heat shock protein 90 \\
\hline \multirow[t]{11}{*}{$\begin{array}{l}\text { DEAD-box ATP-dependent } \\
\text { RNA helicase }\end{array}$} & CL4257.Contig4_S2_1 & 2.38 & Up & $3.15 \mathrm{E}-24$ & $1.95 \mathrm{E}-22$ & $\begin{array}{l}\text { DEAD-box ATP-dependent } \\
\text { RNA helicase } 56 \text {-like }\end{array}$ \\
\hline & Unigene6176_S2_3 & 2.29 & Up & $6.45 \mathrm{E}-22$ & $3.62 \mathrm{E}-20$ & $\begin{array}{l}\text { DEAD-box ATP-dependent } \\
\text { RNA helicase 32-like }\end{array}$ \\
\hline & CL8809.Contig2_S2_3 & 2.17 & Up & 4.76E-07 & 8.64E-06 & $\begin{array}{l}\text { DEAD-box ATP-dependent } \\
\text { RNA helicase 26-like }\end{array}$ \\
\hline & CL4129.Contig1_S2_1 & 1.82 & Up & $4.02 \mathrm{E}-122$ & 1.30E-119 & $\begin{array}{l}\text { DEAD-box ATP-dependent } \\
\text { RNA helicase } 31 \text {-like }\end{array}$ \\
\hline & CL869.Contig2_S2_1 & 1.45 & Up & $3.01 \mathrm{E}-38$ & $3.03 E-36$ & $\begin{array}{l}\text { DEAD-box ATP-dependent } \\
\text { RNA helicase } 28 \text {-like }\end{array}$ \\
\hline & Unigene233_S2_1 & 1.21 & Up & $1.32 \mathrm{E}-07$ & $2.59 \mathrm{E}-06$ & $\begin{array}{l}\text { dead box ATP-dependent } \\
\text { RNA helicase, putative }\end{array}$ \\
\hline & Unigene73562_S2_1 & 1.21 & Up & $6.32 \mathrm{E}-46$ & 7.85E-44 & $\begin{array}{l}\text { DEAD-box ATP-dependent } \\
\text { RNA helicase } 47 \text {, mitochondrial }\end{array}$ \\
\hline & CL6411.Contig2_S2_1 & 1.12 & Up & $1.06 \mathrm{E}-05$ & 0.000155014 & $\begin{array}{l}\text { dead box ATP-dependent } \\
\text { RNA helicase, putative }\end{array}$ \\
\hline & Unigene27627_S2_3 & 1.05 & Up & $2.71 \mathrm{E}-07$ & $5.10 \mathrm{E}-06$ & $\begin{array}{l}\text { DEAD-box ATP-dependent } \\
\text { RNA helicase 24-like }\end{array}$ \\
\hline & Unigene14283_S2_1 & 1.04 & Up & $1.55 E-21$ & $8.56 \mathrm{E}-20$ & $\begin{array}{l}\text { DEAD-box ATP-dependent } \\
\text { RNA helicase 21-like }\end{array}$ \\
\hline & Unigene97540_S2_3 & 1.04 & Up & 4.90E-09 & 1.13E-07 & $\begin{array}{l}\text { DEAD-box ATP-dependent } \\
\text { RNA helicase } 50 \text {-like }\end{array}$ \\
\hline
\end{tabular}

HSP90-encoding DTGs (all up-regulated) featured in the CKA, CKC1 and CKC2 treatments. HSP90s function as molecular chaperones during signal transduction, cell cycling, the stress response, and in protein folding, degradation and transport $[67,68]$. In A. thaliana, expression of Hsp90 is developmentally regulated, but it also responds to high and low temperature, as well as salinity [67]. It is speculated that Hsp90 as molecular chaperones play an important role in signal transduction and stress management in C. nankinginse. A gene HSP70- encoding DTGs (all down-regulated) featured in the CKA, CKB2, CKC1 and CKC2 treatments, which not found in the CKB1 treatment; and a number of small HSPs, belonging to the 15.7, 22.7 and 23.6 families were also among the DTGs identified in treatments involving low temperature response.

In conclusion, it was clear that a large number of genes were induced when exposed to low temperature. The complex gene networks involved a set of interactions 


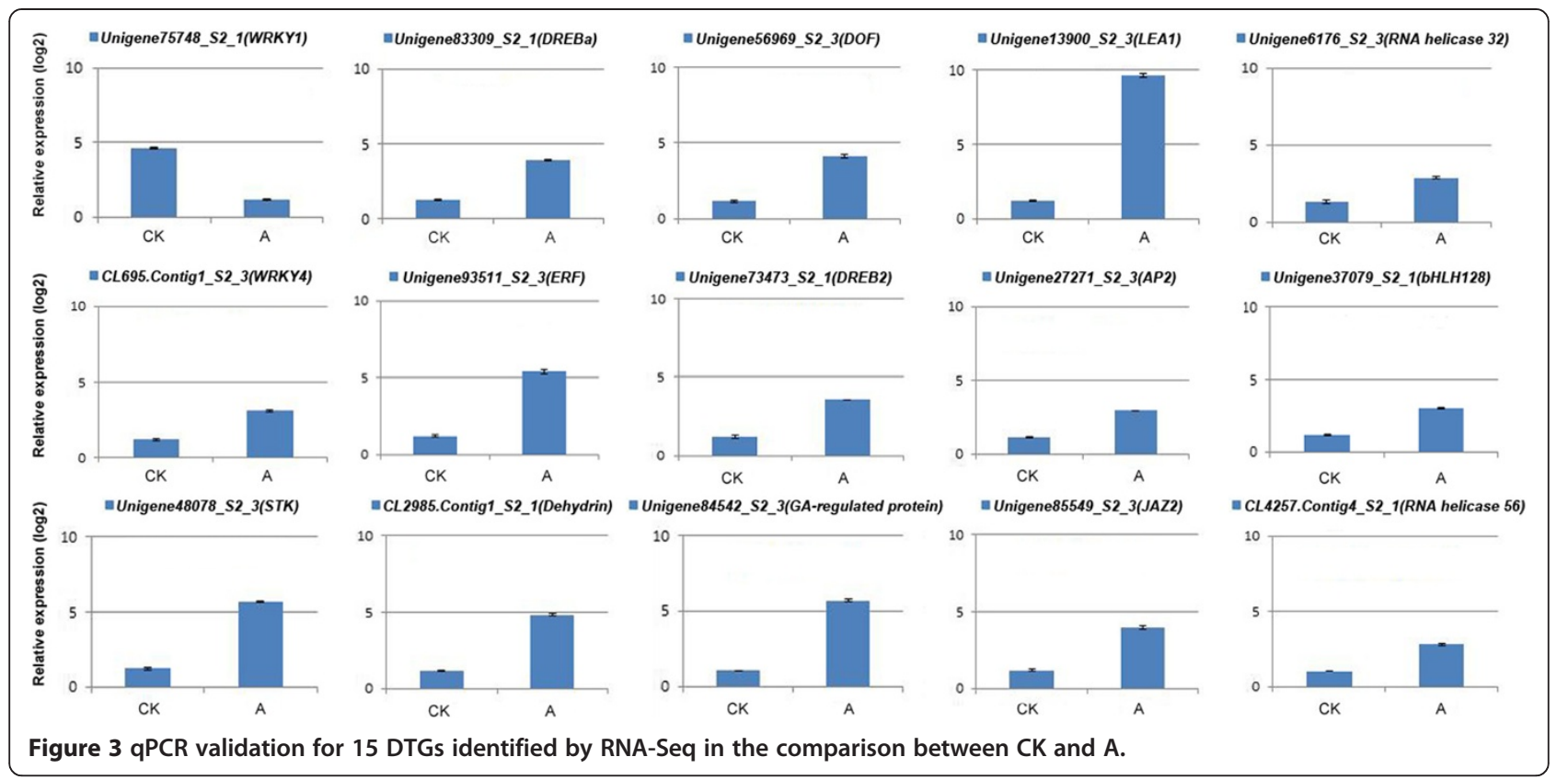

between cold sensing and signaling genes, genes related to post-transcription regulation responding to cold stress, transcription factors and certain functional proteins. In the present study, the DTGs identified as candidate genes in response to low temperature, require further investigation for a complete understanding of the molecular basis of cold response in $C$. nankingense. This information will prove beneficial in molecular breeding programs for excellent chrysanthemum varieties.

\section{Conclusions}

An overview of the many changes to the C. nankingense transcriptome induced by exposure to low temperature has been provided. Many of the DTGs identified were involved in predictable classes of gene, encoding, for example, low temperature sensors and signaling molecules, TFs and certain functional proteins associated with the stress response. The large number of DTGs identified confirms the complexity of the regulatory machinery involved in low temperature acclimation and low temperature/freezing tolerance. Establishing which of these many DTGs reflect the primary response to low temperature is necessary before the molecular basis of the response can be fully elaborated; such genes would represent candidates for intervention in the breeding of chrysanthemum varieties endowed with a heightened tolerance to low temperature stress.

\section{Methods}

\section{Plant material}

The accession of C.nankingense utilized is conserved by the Chrysanthemum Germplasm Resource Preserving Centre,
Nanjing Agricultural University, China. Plants raised from tissue-cultured plantlet were grown on MS medium (16 h photoperiod, $22^{\circ} \mathrm{C} / 18^{\circ} \mathrm{C}$ day/night temperature, $70 \%$ relative humidity). Four week old plants were subjected to one of the following temperature treatments: (A) $4^{\circ} \mathrm{C}$ for one week, (B1) $-5^{\circ} \mathrm{C}$ for $1 \mathrm{~h},(\mathrm{~B} 2)-5^{\circ} \mathrm{C}$ for $2 \mathrm{~h},(\mathrm{C} 1) 4^{\circ} \mathrm{C}$ for one week, followed by $-5^{\circ} \mathrm{C}$ for $1 \mathrm{~h},(\mathrm{C} 2) 4^{\circ} \mathrm{C}$ for one week, followed by $-5^{\circ} \mathrm{C}$ for $2 \mathrm{~h}$. CK plants were harvested without any additional treatment. For each treatment, the leaves and stems of three seedlings were sampled, and two samples were harvested. $30 \mu \mathrm{g}$ of total RNA were pooled in equal amounts from the two biological replicates for subsequent RNA- Seq.

\section{RNA isolation and CDNA library construction}

Six separate libraries were prepared. The samples from the six treatments (A, B1, B2, C1, C2 and CK) were snap-frozen in liquid nitrogen and ground to a fine powder. Total RNA was extracted using a Total RNA Isolation System (Takara, Japan) according to the manufacturer's instructions. When the quality of the resulting RNA was verified using a 2100 Bioanalyzer RNA Nano chip device (Agilent, Santa Clara, CA, USA), all six extractions delivered an RNA integrity number value of $>$ 8.0 , and a $28 \mathrm{~S}: 18 \mathrm{~S}$ ratio $>1.5$. After checking for the absence of contamination by protein (A260/A280 $\mathrm{nm}$ ratios) and reagent contamination (A260/A230 $\mathrm{nm}$ ratios) by a Nanodrop ND-430 1000 spectrophotometer, the extractions were selected based on 28S/18S rRNA band intensity (1.5:1 2:1) and spectroscopic A260/A280 nm readings between 1.8 and 2.2, A260/A230 $\mathrm{nm}$ readings greater than 2.0. $10 \mu \mathrm{g}$ RNA was pooled from each of the three 
sampled plants. The total RNA preparation was treated with RNase-free DNase I (Takara, Japan) to degrade any possible DNA, and mixed with oligo (dT) coated magnetic beads to concentrate the polyA mRNA.

The mRNA was fragmented into short fragments $\sim 200 \mathrm{nt}$ pieces by incubation in a fragmentation buffer under elevated temperature (The Beijing Genomics Institute). The first strand of cDNA was then synthesized by priming with random hexamer, and the second strand was generated with buffer, dNTPs, RNase $\mathrm{H}$ and DNA polymerase I. The double strand cDNA was purified with a QiaQuick PCR extraction kit and resolved with EB buffer for end repair and addition of single nucleotide A. Finally, sequencing adaptors were ligated to the fragments. Following agarose gel electrophore, suitable fragments were selected as templates for PCR.

\section{RNA-Seq}

The library was sequenced using an Illumina HiSeq 2000 located at the Beijing Genomics Institute (Shenzhen, China; http://www.genomics.cn/index). The data were deposited in the US National Center for Biotechnology Information (NCBI) Sequence Read Archive (SRA, http://www. ncbi.nlm.nih.gov/Traces/sra; [69] under accession number (SRP041138). Raw data were saved as .fastq files. Data filtering is performed to obtain "clean reads" for further analysis. Clean reads were obtained by removing adaptor sequences, reads in which the percentage of unknown bases $(\mathrm{N})$ was greater than $10 \%$ and low quality reads. The clean reads were mapped onto the reference sequences using SOAP (2.21) software [70]. A maximum of two mismatches was allowed in the alignment. The NCBI nonredundant protein $(\mathrm{Nr})$ database (http://www.ncbi.nlm.nih. gov) and the Swiss-Prot protein database (http://www. expasy.ch/sprot) were used for blast search and annotation using an $E$-value cut off of $10^{-5}$. Functional annotation by gene ontology terms (GO, http://www. geneontology.org) was analyzed using the Blast2GO program [71]. The Kyoto Encyclopedia of Genes and Genomes Pathway (KEGG; http://www.genome.jp/kegg), the major public pathway-related database [72] was also used to predict and classify possible functions. The RPKM reads (clean reads per kilo base per million) method [31] was used to estimate transcript abundance on the base of eliminating the influence of different gene length and sequencing discrepancy. Therefore, the RPKM values can be directly used for comparing the difference of gene expression among samples.

\section{Identification of differentially expressed genes}

To compare the differences in gene expression, the method of an algorithm developed by Audic et al. [32] was used to identify DTGs. The criteria applied were an FDR (false discovery rate) less than 0.01 and an absolute value of $\log 2$ ratio of at least 1 . Then, the DTGs were subjected to GO and KEGG Ontology (KO) enrichment analysis on the base of a hypergeometric test.

\section{qPCR validation of differential transcription}

Total RNA was isolated from leave and stem of plants subjected to the various treatments described above. Contaminating DNA was removed by treating with RNasefree DNase I and the first cDNA strand was synthesized from $1 \mu \mathrm{g}$ total RNA using PrimeScript ${ }^{\ominus}$ Reverse Transcriptase (Takara, Dalian, China) and an oligo (dT) primer, according to the manufacturer's instructions. qPCRs were

Table 7 Primers of quantitative reverse transcription-polymerase chain reaction for validation of RNA-Seq data

\begin{tabular}{|c|c|c|c|}
\hline GenelD & Primer F (5'-3') & Primer R (5'-3') & Blast $\mathrm{nr}$ \\
\hline CL695.Contig1_S2_3 & GTGACGAGTTGGTGATGGTG & GTTACCACCTACGAAGGCCA & WRKY transcription factor 4 \\
\hline Unigene75748_S2_1 & CGGGTGAAATGCTCTCAAAT & TGCCAAATGGTTCTAAAGGG & WRKY transcription factor 1 \\
\hline Unigene83309_S2_1 & ATTTAAACACGCGGATCGAC & CCAGAGTGTGGCTTGGTACA & DREBa \\
\hline Unigene73473_S2_1 & TAAAGGTGGGCCAGAAAATG & ATCATACGCCAGAGCAGCTT & DREB2 transcription factor \\
\hline Unigene27271_S2_3 & ACAACATCCCCTTGGATGAA & GGGTGACAGCATTTGAAGGT & AP2 transcription factor \\
\hline Unigene93511_S2_3 & TGTGCCGCTGTTATCCATTA & CCACACTATCACAGCCCCTT & Ethylene-responsive transcription factor \\
\hline Unigene37079_S2_1 & TCTTCTTTCCCTTTCTGCGA & TGGATCTCCCTCATGACTCC & bHLH128-like \\
\hline Unigene56969_S2_3 & GCATTTGCAGCTGATTCTGA & GCTATCACCGTTGACCCACT & DOF domain class transcription factor \\
\hline Unigene13900_S2_3 & ATCGTGTCGCCGGTATTTAG & GTTGTAGACAAAGCGTCGCA & LEA protein 1 \\
\hline CL2985.Contig1_S2_1 & CATCCCCATATTGGTTCCAG & GAACACGAAGCAAGAGGGTC & dehydrin \\
\hline CL4257.Contig4_S2_1 & CTTCTTGCACACTGGTCGAA & GGGGCTTGCTAGGGATAAAG & DEAD-box ATP-dependent RNA helicase 56-like \\
\hline Unigene6176_S2_3 & TGTTTGGCTTGTCAAACTGG & TCCGTGTTATTCCTITTGCC & DEAD-box ATP-dependent RNA helicase 32-like \\
\hline Unigene84542_S2_3 & CCAGGTTTCGTTITCGTCAT & GCCTTGAATGCTTTCCACAT & Gibberellin-regulated protein \\
\hline Unigene85549_S2_3 & ACCTCTGTCGGTCCATCAAC & TCGGAACGAGCTCATCTITT & jasmonate ZIM-domain protein 2 \\
\hline Unigene48078_S2_3 & TTTCAGCCGATGGTGATGTA & GTCGTGCCCCACAAGATACT & Serine/threonine-protein kinase \\
\hline
\end{tabular}


performed in an Eppendorf Real Time PCR System (Mastercycler ${ }^{\circ}$ ep realplex, Germany) using a SYBR Premix Ex $\mathrm{Taq}^{\mathrm{Tm}}$ Kit (Takara), according to the manufacturer's protocol. Gene-specific primers were designed using Primer5 software (sequences given in Table 7). Each $20 \mu \mathrm{L}$ qPCR contained $5 \mu \mathrm{L}$ diluted cDNA, $100 \mathrm{nM}$ of each primer, and $10 \mu \mathrm{l} \mathrm{SYBR}$ Green PCR master mix, and was exposed to an initial denaturation $\left(95^{\circ} \mathrm{C} / 2 \mathrm{~min}\right)$, followed by $40 \mathrm{cy}$ cles of $95^{\circ} \mathrm{C} / 15 \mathrm{~s}, 60^{\circ} \mathrm{C} / 15 \mathrm{~s}, 72^{\circ} \mathrm{C} / 15 \mathrm{~s}$. After amplication, all results were screened to verify a single peak melting curve for the specificity of the amplifications. Three biological replicates were performed for each sample. Relative transcript abundance was obtained by including the C. nankingense EF1 $\alpha$ gene as the reference, and was based on the $2^{-\Delta \Delta C T}$ method [73].

\section{Additional files}

Additional file 1: Figure S1. Composition of raw reads in the six RNA libraries. "Clean" reads refers to those remaining after the removal of adaptor sequences, reads in which the proportion of missing bases was $>10 \%$ and reads in which low quality $(\leq 5)$ bases represented $>50 \%$ of the reads. The numbers in parentheses indicate the percentage of each type of read present.

Additional file 2: Figure S2. Sequencing saturation analysis in the six libraries (A, B1, B2, C1, C2 and CK). The numbers of new genes detected rose as the read number was increased, but not beyond a threshold around 7,000,000.

Additional file 3: Figure S3. Distribution of gene coverage in the six libraries.

Additional file 4: Table S1. The transcription level of each unigene derived from the number of relevant reads recovered in the four libraries. The "GeneLength" column gives the length of exon sequence.

Additional file 5: Table S2. Genes differentially transcribed in the comparison between libraries CK and A. The criteria applied for assigning significance were: P-value $<0.05, F D R \leq 0.001$, and estimated absolute $\left|\log 2^{\text {Ratio(A/CK) }}\right| \geq 1$. Genes listed in descending order of absolute $\mid \log 2^{\text {Ratio(A/CK) }}$. GenelDs retrieved from the Chrysanthemum nankingense Reference Sequence Database. Annotation of unigene sequences performed using BlastX $(E<10)$. The "GeneLength" column gives the length of exon sequence. CK- and A- expression: frequency of unigene transcripts in libraries CK and A, respectively. CK- and A-RPKM: reads per kb per million reads for each unigene in libraries $C K$ and $A$, respectively. $\log 2^{\text {Ratio( }(A / C K)}$ : the ratio between the RPKM in CK and the RPKM in A. Up-Down-Regulation (A/CK), $P$-value and FDR of each gene are also shown. KEGG: annotation according to the KEGG database by BLAST. Blast nr: identification of homologues in GenBank. GO Component, GO Function and Go Process: ontology information of Cellular Components, Molecular Function and Biological Processes of Gene-corresponding GO terms. "-": no hit.

Additional file 6: Table S3. Genes differentially transcribed in the comparison between libraries CK and B1. The criteria applied for assigning significance were: P-value $<0.05, F D R \leq 0.001$, and estimated absolute $\left|\log 2^{\text {Ratio(B1/CK) }}\right| \geq 1$. Genes listed in descending order of absolute $\left|\log 2^{\text {Ratio(B1/CK)}}\right|$. GenelDs retrieved from Chrysanthemum nankingense Reference Sequence Database. Annotation of unigene sequences performed using BlastX ( $E<10$ ). The "GeneLength" column gives the length of exon sequence. CK- and B1-expression: frequency of unigene transcripts in libraries CK and B1, respectively. CK- and B1-RPKM: reads per $\mathrm{kb}$ per million reads for each unigene in libraries CK and B1, respectively. $\log 2^{\text {Ratio(B1/CK): }}$ the ratio between the RPKM in CK and the RPKM in B1. Up-Down-Regulation (B1/CK), P-value and FDR of each gene are also shown. KEGG: annotation according to the KEGG database by BLAST. Blast nr: identification of homologues in GenBank. GO Component, GO
Function and Go Process: ontology information of Cellular Components, Molecular Function and Biological Processes of Gene-corresponding GO terms. "-": no hit.

Additional file 7: Table S4. Genes differentially transcribed in the comparison between libraries CK and B2. The criteria applied for assigning significance were: P-value $<0.05, F D R \leq 0.001$, and estimated absolute $\left|\log 2^{\text {Ratio(B2/CK) }}\right| \geq 1$. Genes listed in descending order of absolute $\left|\log 2^{\text {Ratio(B2/CK) }}\right|$. GenelDs retrieved from the Chrysanthemum nankingense Reference Sequence Database. Annotation of unigene sequences performed using BlastX ( $E<10$ ). The "GeneLength" column gives the length of exon sequence. CK- and B2-expression: frequency of unigene transcripts in libraries CK and B2, respectively. CK- and B2-RPKM: reads per $\mathrm{kb}$ per million reads for each unigene in libraries $C K$ and $B 2$, respectively.

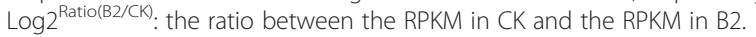
Up-Down-Regulation (B2/CK), P-value and FDR of each gene are also shown. KEGG: annotation according to the KEGG database by BLAST. Blast nr: identification of homologues in GenBank. GO Component, GO Function and Go Process: ontology information of Cellular Components, Molecular Function and Biological Processes of Gene-corresponding GO terms. "-": no hit.

Additional file 8: Table S5. Genes differentially transcribed in the comparison between libraries CK and C1. The criteria applied for assigning significance were: P-value $<0.05, F D R \leq 0.001$, and estimated absolute $\left|\log 2^{\text {Ratio( }(1 / C K)}\right| \geq 1$. Genes listed in descending order of absolute $\left|\log 2^{\text {Ratio( }(\mathrm{C} / \mathrm{CK})}\right|$. GenelDs retrieved from the Chrysanthemum nankingense Reference Sequence Database. Annotation of unigene sequences performed using BlastX ( $E<10)$. The "GeneLength" column gives the length of exon sequence. CK- and C1-expression: frequency of unigene transcripts in libraries CK and C1, respectively. CK- and C1-RPKM: reads per $\mathrm{kb}$ per million reads for each unigene in libraries $C K$ and $C 1$, respectively. Log $2^{\text {Ratio( } C 1 / C K)}$ : the ratio between the RPKM in CK and the RPKM in C1. Up-Down-Regulation (C1/CK), P-value and FDR of each gene are also shown. KEGG: annotation according to the KEGG database by BLAST. Blast nr: identification of homologues in GenBank. GO Component, GO Function and Go Process: ontology information of Cellular Components, Molecular Function and Biological Processes of Gene-corresponding GO terms. "-": no hit.

Additional file 9: Table S6. Genes differentially transcribed in the comparison between libraries CK and C2. The criteria applied for assigning significance were: P-value $<0.05, F D R \leq 0.001$, and estimated absolute $\left|\log 2^{\text {Ratio(C2/CK) }}\right| \geq 1$. Genes listed in descending order of absolute | $\log 2^{\text {Ratio( }(\mathrm{C} / \mathrm{CK})} \mid$. GenelDs retrieved from the Chrysanthemum nankingense Reference Sequence Database. Annotation of unigene sequences performed using BlastX $(E<10)$. The "GeneLength" column gives the length of exon sequence. CK- and C2-expression: frequency of unigene transcripts in libraries CK and C2, respectively. CK- and C2-RPKM: reads per kb per million reads for each unigene in libraries CK and C2, respectively. Log $2^{\text {Ratio }}$ $(\mathrm{C} 2 / \mathrm{CK})$ : the ratio between the RPKM in CK and the RPKM in C2. Up-DownRegulation (C2/CK), P-value and FDR of each gene are also shown. KEGG: annotation according to the KEGG database by BLAST. Blast nr: identification of homologues in GenBank. GO Component, GO Function and Go Process: ontology information of Cellular Components, Molecular Function and Biological Processes of Gene-corresponding GO terms. "-": no hit.

Additional file 10: Table S7. Genes differentially transcribed in the comparison between libraries A and C1. The criteria applied for assigning significance were: P-value $<0.05, F D R \leq 0.001$, and estimated absolute $\left|\log 2^{\text {Ratio(C1/A) }}\right| \geq 1$. Genes listed in descending order of absolute $\left|\log 2^{\text {Ratio(C1/A) }}\right|$. GenelDs retrieved from the Chrysanthemum nankingense Reference Sequence Database. Annotation of unigene sequences performed using BlastX ( $<<10$ ). The "GeneLength" column gives the length of exon sequence. A- and C1-expression: frequency of unigene transcripts in libraries $A$ and $C 1$, respectively. A- and C1-RPKM: reads per $\mathrm{kb}$ per million reads for each unigene in libraries $A$ and $C 1$, respectively. $\log 2^{\text {Ratio( } C 1 / A)}$ : the ratio between the RPKM in A and the RPKM in C1. Up-Down-Regulation (C1/A), P-value and FDR of each gene are also shown. KEGG: annotation according to the KEGG database by BLAST. Blast nr: identification of homologues in GenBank. GO Component, GO Function and Go Process: ontology information of Cellular Components, Molecular Function and Biological Processes of Gene-corresponding GO terms. "-": no hit. 
Additional file 11: Table S8. Genes differentially transcribed in the comparison between libraries A and C2. The criteria applied for assigning significance were: P-value $<0.05, F D R \leq 0.001$, and estimated absolute $\left|\log 2^{\text {Ratio(C2/A) }}\right| \geq 1$. Genes listed in descending order of absolute $\left|\log 2^{\text {Ratio(C2/A } \mid}\right|$. GenelDs retrieved from the Chrysanthemum nankingense Reference Sequence Database. Annotation of unigene sequences performed using BlastX ( $E<10$ ). The "GeneLength" column gives the length of exon sequence. A- and C2-expression: frequency of unigene transcripts in libraries A and C2, respectively. A- and C2-RPKM: reads per $\mathrm{kb}$ per million reads for each unigene in libraries $A$ and $C 2$, respectively. $\log _{2}{ }^{\text {Ratio(C2/A) }}$ : the ratio between the RPKM in A and the RPKM in C2. Up-Down-Regulation (C2/A), P-value and FDR of each gene are also shown. KEGG: annotation according to the KEGG database by BLAST. Blast nr: identification of homologues in GenBank. GO Component, GO Function and Go Process: ontology information of Cellular Components, Molecular Function and Biological Processes of Gene-corresponding GO terms. "-": no hit.

Additional file 12: Table S9. Expression pattern analysis of DTGS following multiple comparisons: CK vs A, CK vs C1, CK vs C2. GenelDs retrieved from the Chrysanthemum nankingense Reference Sequence Database. Log2 Ratio(A)CK): the ratio between the RPKM in CK and the RPKM in A. Log $2^{\text {Ratio( }(1 / C K)}$ : the ratio between the RPKM in CK and the RPKM in

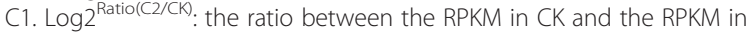
C2. KEGG: annotation according to the KEGG database by BLAST. Blast nr: identification of homologues in GenBank. GO Component, GO Function and Go Process: ontology information of Cellular Components, Molecular Function and Biological Processes of Gene-corresponding GO terms. "-": no hit.

Additional file 13: Table S10. Expression pattern analysis of DTGs following multiple comparisons: CK vs B1, CK vs B2. GenelDs retrieved from the Chrysanthemum nankingense Reference Sequence Database.

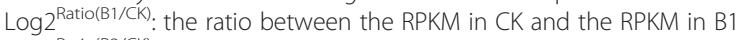

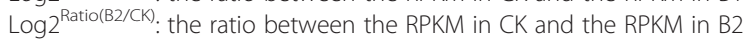
KEGG: annotation according to the KEGG database by BLAST. Blast nr: identification of homologues in GenBank. GO Component, GO Function and Go Process: ontology information of Cellular Components, Molecular Function and Biological Processes of Gene-corresponding GO terms. "-": no hit.

Additional file 14: Table S11. Expression pattern analysis of DTGS following multiple comparisons: A vs C1, A vs C2. GenelDs retrieved from the Chrysanthemum nankingense Reference Sequence Database. Log2 Ratio(C1/A): the ratio between the RPKM in A and the RPKM in C1. $\log _{2} 2^{\operatorname{Ratio}(C 2 / A)}$ : the ratio between the RPKM in A and the RPKM in C2. KEGG: annotation according to the KEGG database by BLAST. Blast nr: identification of homologues in GenBank. GO Component, GO Function and Go Process: ontology information of Cellular Components, Molecular Function and Biological Processes of Gene-corresponding GO terms. "-": no hit.

Additional file 15: Table S12. GO classification of DTGs in each comparison.

Additional file 16: Table S13. Pathway classification of DTGs in each comparison.

\section{Competing interests}

The authors declare that they have no competing interests.

\section{Authors' contributions}

RLP and SJ contributed to bioinformatics analysis and writing of the manuscript. JJF, CFD, CSM, and TNJ conceived of the study, participated in its design and contributed to revisions of the manuscript. LY, FWM and GZY participated in experiment materials preparation. GJJ, DB and WYJ helped with the RNA extraction. XXL and LYN contributed to real-time PCR, all authors read and approved the final manuscript.

\section{Acknowledgment}

This work was supported by funding from the Program for New Century Excellent Talents in University of Chinese Ministry of Education (NCET-10-0492, NCET-12-0890, NCET-11-0669), the National Natural Science Foundation of China $(31272196,31171987,31372100)$, the Natural Science Fund of Jiangsu Province (BK201164, BK2012773), 948 Project of Ministry of Agriculture (2011-G17, 2013-S13), Fund for Independent Innovation of Agricultural Sciences in Jiangsu Province [CX(12)2020], the Program for Hi-Tech Research, Jiangsu,
China, (BE2012350,BE2011325), Fundamental Research Funds for the Central Universities (KYZ201 147), and the Qing Lan Project of Jiangsu Province.

Received: 12 May 2014 Accepted: 23 September 2014 Published: 3 October 2014

\section{References}

1. Liu L, Zhu K, Yang Y, Wu J, Chen F, Yu D: Molecular cloning, expression profiling and trans-activation property studies of a DREB2-like gene from chrysanthemum (Dendranthema vestitum). J Plant Res 2008, 121(2):215-226.

2. da Silva JA T, Shinoyama H, Aida R, Matsushita Y, Raj SK, Chen F: Chrysanthemum biotechnology: Quo Vadis? Crit Rev Plant Sci 2013, 32(1):21-52.

3. Cheng X, Chen S, Chen F, Fang W, Deng Y, She L: Interspecific hybrids between Dendranthema morifolium (Ramat.) Kitamura and $D$. nankingense (Nakai) Tzvel. achieved using ovary rescue and their cold tolerance characteristics. Euphytica 2009, 172(1):101-108.

4. Wang H, Jiang J, Chen S, Qi X, Peng H, Li P, Song A, Guan Z, Fang W, Liao $Y$ : Next-generation sequencing of the Chrysanthemum nankingense (Asteraceae) transcriptome permits large-scale unigene assembly and SSR marker discovery. PLoS One 2013, 8(4):e62293.

5. Janská A, Maršík P, Zelenková S, Ovesná J: Cold stress and acclimationwhat is important for metabolic adjustment? Plant Biol 2010, 12(3):395-405.

6. Yang T, Zhang L, Zhang T, Zhang H, Xu S, An L: Transcriptional regulation network of cold-responsive genes in higher plants. Plant Sci 2005, 169(6):987-995.

7. Chinnusamy V, Zhu J, Zhu J-K: Cold stress regulation of gene expression in plants. Trends Plant Sci 2007, 12(10):444-451.

8. Chinnusamy V, Zhu JK, Sunkar R: Gene regulation during cold stress acclimation in plants. Plant Stress Tolerance 2010, 639:39-55.

9. Yamaguchi-Shinozaki K, Shinozaki K: Transcriptional regulatory networks in cellular responses and tolerance to dehydration and cold stresses. Annu Rev Plant Biol 2006, 57:781-803.

10. Heidarvand $L$, Amiri RM: What happens in plant molecular responses to cold stress? Acta Physiol Plant 2010, 32(3):419-431.

11. Bohn M, Lüthje S, Sperling P, Heinz E, Dörffling K: Plasma membrane lipid alterations induced by cold acclimation and abscisic acid treatment of winter wheat seedlings differing in frost resistance. J Plant Physiol 2007, 164(2):146-156.

12. Lynch DV, Steponkus PL: Plasma membrane lipid alterations associated with cold acclimation of winter rye seedlings (Secale cereale L. cv Puma). Plant Physiol 1987, 83(4):761-767.

13. Uemura M, Joseph RA, Steponkus PL: Cold acclimation of Arabidopsis thaliana (effect on plasma membrane lipid composition and freezeinduced lesions). Plant Physiol 1995, 109(1):15-30.

14. Uemura M, Tominaga Y, Nakagawara C, Shigematsu S, Minami A, Kawamura $Y$ : Responses of the plasma membrane to low temperatures. Physiol Plant 2006, 126(1):81-89.

15. Komatsu S, Yang G, Khan M, Onodera H, Toki S, Yamaguchi M: Overexpression of calcium-dependent protein kinase 13 and calreticulin interacting protein 1 confers cold tolerance on rice plants. Mol Gen Genomics 2007, 277(6):713-723.

16. Saijo Y, Hata S, Kyozuka J, Shimamoto K, Izui K: Over-expression of a single $\mathrm{Ca}^{2+}$-dependent protein kinase confers both cold and salt/drought tolerance on rice plants. Plant J 2000, 23(3):319-327.

17. Lissarre M, Ohta M, Sato A, Miura K: Cold-responsive gene regulation during cold acclimation in plants. Plant Signal Behav 2010, 5(8):948-952.

18. Yang G, Zou H, Wu Y, Liu H, Yuan Y: Identification and characterisation of candidate genes involved in chilling responses in maize (Zea mays L.). Plant Cell, Tissue and Organ Culture (PCTOC) 2011, 106(1):127-141.

19. Thomashow MF: Molecular basis of plant cold acclimation: insights gained from studying the CBF cold response pathway. Plant Physiol 2010, 154(2):571-577.

20. Dong MA, Farré EM, Thomashow MF: Circadian clock-associated 1 and late elongated hypocotyl regulate expression of the C-repeat binding factor (CBF) pathway in Arabidopsis. Proc Natl Acad Sci 2011, 108(17):7241-7246.

21. B-h L, Henderson DA, Zhu J-K: The Arabidopsis cold-responsive transcriptome and its regulation by ICE1. Plant Cell Online 2005, 17(11):3155-3175. 
22. Dong $C-H$, Agarwal M, Zhang $Y$, Xie Q, Zhu J-K: The negative regulator of plant cold responses, HOS1, is a RING E3 ligase that mediates the ubiquitination and degradation of ICE1. Proc Natl Acad Sci 2006, 103(21):8281-8286.

23. Miura K, Ohta M: SIZ1, a small ubiquitin-related modifier ligase, controls cold signaling through regulation of salicylic acid accumulation. J Plant Physiol 2010, 167(7):555-560.

24. Scott IM, Clarke SM, Wood JE, Mur LA: Salicylate accumulation inhibits growth at chilling temperature in Arabidopsis. Plant Physiol 2004, 135(2):1040-1049.

25. Zhu J, Dong C-H, Zhu J-K: Interplay between cold-responsive gene regulation, metabolism and RNA processing during plant cold acclimation. Curr Opin Plant Biol 2007, 10(3):290-295.

26. Ma H, Lu Z, Liu B, Qiu Q, Liu J: Transcriptome analyses of a Chinese hazelnut species Corylus mandshurica. BMC Plant Biol 2013, 13(1):152

27. Hornett EA, Wheat CW: Quantitative RNA-Seq analysis in non-model species: assessing transcriptome assemblies as a scaffold and the utility of evolutionary divergent genomic reference species. BMC Genomics 2012, 13(1):361.

28. Wang X-C, Zhao Q-Y, Ma C-L, Zhang Z-H, Cao H-L, Kong Y-M, Yue C, Hao $X-Y$, Chen L, Ma J-Q: Global transcriptome profiles of Camellia sinensis during cold acclimation. BMC Genomics 2013, 14(1):415.

29. Wang H, Zou Z, Wang S, Gong M: Global analysis of transcriptome responses and gene expression profiles to cold stress of Jatropha curcas L. PLoS One 2013, 8(12):e82817.

30. Tian D-Q, Pan X-Y, Yu Y-M, Wang W-Y, Zhang F, Ge Y-Y, Shen X-L, Shen F-Q, Liu X-J: De novo characterization of the Anthurium transcriptome and analysis of its digital gene expression under cold stress. BMC Genomics 2013, 14(1):827.

31. Mortazavi A, Williams BA, McCue K, Schaeffer L, Wold B: Mapping and quantifying mammalian transcriptomes by RNA-Seq. Nat Methods 2008, 5(7):621-628.

32. Audic S, Claverie J-M: The significance of digital gene expression profiles. Genome Res 1997, 7(10):986-995.

33. $X u L$, Zhu L, Tu L, Liu L, Yuan D, Jin L, Long L, Zhang X: Lignin metabolism has a central role in the resistance of cotton to the wilt fungus Verticillium dahliae as revealed by RNA-Seq-dependent transcriptional analysis and histochemistry. J Exp Bot 2011, 62(15):5607-5621.

34. Reddy AS, Ali GS, Celesnik H, Day IS: Coping with stresses: roles of calcium-and calcium/calmodulin-regulated gene expression. Plant Cell Online 2011, 23(6):2010-2032.

35. Miura K, Furumoto T: Cold signaling and cold response in plants. Int J Mol Sci 2013, 14(3):5312-5337.

36. Boudsocq $M$, Sheen J: CDPKs in immune and stress signaling. Trends Plant Sci 2013, 18(1):30-40.

37. Morris ER, Walker JC: Receptor-like protein kinases: the keys to response. Curr Opin Plant Biol 2003, 6(4):339-342.

38. Lehti-Shiu MD, Zou C, Hanada K, Shiu S-H: Evolutionary history and stress regulation of plant receptor-like kinase/pelle genes. Plant Physiol 2009, 150(1):12-26.

39. McCormack E, Braam J: Calmodulins and related potential calcium sensors of Arabidopsis. New Phytol 2003, 159(3):585-598.

40. Xu G-Y, Rocha PS, Wang M-L, Xu M-L, Cui Y-C, Li L-Y, Zhu Y-X, Xia X: A novel rice calmodulin-like gene, OSMSR2, enhances drought and salt tolerance and increases ABA sensitivity in Arabidopsis. Planta 2011, 234(1):47-59.

41. Hashimoto K, Kudla J: Calcium decoding mechanisms in plants. Biochimie 2011, 93(12):2054-2059.

42. Schulz $P$, Herde M, Romeis T: Calcium-dependent protein kinases: hubs in plant stress signaling and development. Plant Physiol 2013, 163(2):523-530.

43. Chen J, Xue B, Xia X, Yin W: A novel calcium-dependent protein kinase gene from Populus euphratica, confers both drought and cold stress tolerance. Biochem Biophys Res Commun 2013, 441(3):630-636.

44. Singh KB, Foley RC, Oñate-Sánchez L: Transcription factors in plant defense and stress responses. Curr Opin Plant Biol 2002, 5(5):430-436.

45. Mizoi J, Shinozaki K, Yamaguchi Shinozaki K: AP2/ERF family transcription factors in plant abiotic stress responses. Biochim Biophysica Acta (BBA)-Gene Regulatory Mechanisms 2012, 1819(2):86-96.

46. Sakuma Y, Liu Q, Dubouzet JG, Abe H, Shinozaki K, Yamaguchi-Shinozaki K. DNA-binding specificity of the ERF/AP2 domain of Arabidopsis DREBs, transcription factors involved in dehydration-and cold-inducible gene expression. Biochem Biophys Res Commun 2002, 290(3):998-1009.

47. Li X, Zhang D, Li H, Wang Y, Zhang Y, Wood AJ: EsDREB2B, a novel truncated DREB2-type transcription factor in the desert legume Eremosparton songoricum, enhances tolerance to multiple abiotic stresses in yeast and transgenic tobacco. BMC Plant Biol 2014, 14(1):44

48. Szabados L, Savouré A: Proline: a multifunctional amino acid. Trends Plant Sci 2010, 15(2):89-97.

49. Kang H-G, Kim J, Kim B, Jeong H, Choi SH, Kim EK, Lee H-Y, Lim PO: Overexpression of $F T L 1 / D D F 1$, an AP2 transcription factor, enhances tolerance to cold, drought, and heat stresses in Arabidopsis thaliana. Plant Sci 2011, 180(4):634-641.

50. Yokotani N, Sato Y, Tanabe S, Chujo T, Shimizu T, Okada K, Yamane H, Shimono M, Sugano S, Takatsuji H: WRKY76 is a rice transcriptional repressor playing opposite roles in blast disease resistance and cold stress tolerance. J Exp Bot 2013, 64(16):5085-5097.

51. H-h P, Shan W, Kuang J-f, Lu W-j, Chen J-y: Molecular characterization of cold-responsive basic helix-loop-helix transcription factors MabHLHs that interact with MaICE1 in banana fruit. Planta 2013, 238(5):937-953.

52. Yang A, Dai X, Zhang W-H: A R2R3-type MYB gene, OsMYB2, is involved in salt, cold, and dehydration tolerance in rice. J Exp Bot 2012, 63(7):2541-2556.

53. Kang H, Park SJ, Kwak KJ: Plant RNA chaperones in stress response. Trends Plant Sci 2012, 18(2):100-106.

54. Fowler S, Thomashow MF: Arabidopsis transcriptome profiling indicates that multiple regulatory pathways are activated during cold acclimation in addition to the CBF cold response pathway. Plant Cell Online 2002, 14(8):1675-1690.

55. Kreps JA, Wu Y, Chang H-S, Zhu T, Wang X, Harper JF: Transcriptome changes for Arabidopsis in response to salt, osmotic, and cold stress. Plant Physiol 2002, 130(4):2129-2141.

56. Lim J, Thomas T, Cavicchioli R: Low temperature regulated DEAD-box RNA helicase from the antarctic archaeon, Methanococcoides burtonii. J Mol Biol 2000, 297(3):553-567.

57. Chamot D, Owttrim GW: Regulation of cold shock-induced RNA helicase gene expression in the cyanobacterium Anabaena sp. strain PCC 7120. J Bacteriol 2000, 182(5):1251-1256.

58. Kim JS, Kim KA, Oh TR, Park CM, Kang H: Functional characterization of DEAD-box RNA helicases in Arabidopsis thaliana under abiotic stress conditions. Plant Cell Physiol 2008, 49(10):1563-1571.

59. Guan Q, Wu J, Zhang Y, Jiang C, Liu R, Chai C, Zhu J: A DEAD box RNA helicase is critical for pre-mRNA splicing, cold-responsive gene regulation, and cold tolerance in Arabidopsis. Plant Cell Online 2013, 25(1):342-356.

60. Asakura Y, Galarneau E, Watkins KP, Barkan A, van Wijk KJ: Chloroplast RH3 DEAD box RNA helicases in maize and Arabidopsis function in splicing of specific group II introns and affect chloroplast ribosome biogenesis. Plant Physiol 2012, 159(3):961-974.

61. Karunatilaka KS, Solem A, Pyle AM, Rueda D: Single-molecule analysis of Mss116-mediated group II intron folding. Nature 2010, 467(7318):935-939.

62. Bhaskaran H, Russell R: Kinetic redistribution of native and misfolded RNAs by a DEAD-box chaperone. Nature 2007, 449(7165):1014-1018.

63. Liu Y, Wang L, Xing X, Sun L, Pan J, Kong X, Zhang M, Li D: ZmLEA3, a multifunctional group 3 LEA protein from maize (Zea mays L.), is involved in biotic and abiotic stresses. Plant Cell Physio/ 2013, 54(6):944-959.

64. Sasaki K, Christov NK, Tsuda S, Imai R: Identification of a novel LEA protein involved in freezing tolerance in wheat. Plant Cell Physiol 2014, 55(1):136-147.

65. Eriksson SK, Kutzer M, Procek J, Gröbner G, Harryson P: Tunable membrane binding of the intrinsically disordered dehydrin Lti30, a cold-induced plant stress protein. Plant Cell Online 2011, 23(6):2391-2404.

66. Fernández M, Valenzuela S: Isolation, characterization and genes expression analysis of three dehydrin genes during cold acclimation of Eucalyptus globulus. BMC Proceedings 2011, 5(Suppl 7):81

67. Wang W, Vinocur B, Shoseyov O, Altman A: Role of plant heat-shock proteins and molecular chaperones in the abiotic stress response. Trends Plant Sci 2004, 9(5):244-252.

68. Chen B, Zhong D, Monteiro A: Comparative genomics and evolution of the HSP90 family of genes across all kingdoms of organisms. BMC Genomics 2006, 7(1):156.

69. Wheeler DL, Barrett T, Benson DA, Bryant SH, Canese K, Chetvernin V, Church DM, DiCuccio M, Edgar R, Federhen S, Geer LY, Helmberg W, Kapustin Y, Kenton DL, Khovayko O, Lipman DJ, Madden TL, Maglott DR, 
Ostell J, Pruitt KD, Schuler GD, Schriml LM, Sequeira E, Sherry ST, Sirotkin K, Souvorov A, Starchenko G, Suzek TO, Tatusov R, Tatusova TA, et al: Database resources of the National Center for Biotechnology Information. Nucleic Acids Res 2008, 36:D13-D21.

70. Li R, Yu C, Li Y, Lam T-W, Yiu S-M, Kristiansen K, Wang J: SOAP2: an improved ultrafast tool for short read alignment. Bioinformatics 2009 25(15):1966-1967.

71. Conesa A, Götz S, García-Gómez JM, Terol J, Talón M, Robles M: Blast2GO: a universal tool for annotation, visualization and analysis in functional genomics research. Bioinformatics 2005, 21(18):3674-3676.

72. Kanehisa M, Araki M, Goto S, Hattori M, Hirakawa M, Itoh M, Katayama T, Kawashima S, Okuda S, Tokimatsu T: KEGG for linking genomes to life and the environment. Nucleic Acids Res 2008, 36(suppl 1):D480-D484.

73. Livak KJ, Schmittgen TD: Analysis of relative gene expression data using real-time quantitative PCR and the $2^{-\Delta \Delta C T}$ Method. Methods 2001, 25(4):402-408.

doi:10.1186/1471-2164-15-844

Cite this article as: Ren et al:: A transcriptomic analysis of

Chrysanthemum nankingense provides insights into the basis of low temperature tolerance. BMC Genomics 2014 15:844.

\section{Submit your next manuscript to BioMed Central and take full advantage of:}

- Convenient online submission

- Thorough peer review

- No space constraints or color figure charges

- Immediate publication on acceptance

- Inclusion in PubMed, CAS, Scopus and Google Scholar

- Research which is freely available for redistribution 